\title{
Basement membrane remodelling regulates mouse embryogenesis
}

https://doi.org/10.1038/s41586-020-2264-2

Received: 14 March 2019

Accepted: 5 March 2020

Published online: 06 May 2020

Check for updates

\author{
Christos Kyprianou ${ }^{1,7}$, Neophytos Christodoulou ${ }^{1,6,7}$, Russell S. Hamilton ${ }^{2,3}$, \\ Wallis Nahaboo ${ }^{4}$, Diana Suarez Boomgaard ${ }^{4}$, Gianluca Amadei ${ }^{1}$, Isabelle Migeotte ${ }^{4}$ \& \\ Magdalena Zernicka-Goetz ${ }^{1,5 凶}$
}

Tissue sculpting during development has been attributed mainly to cellular events through processes such as convergent extension or apical constriction ${ }^{1,2}$. However, recent work has revealed roles for basement membrane remodelling in global tissue morphogenesis ${ }^{3-5}$. Upon implantation, the epiblast and extraembryonic ectoderm of the mouse embryo become enveloped by a basement membrane. Signalling between the basement membrane and these tissues is critical for cell polarization and the ensuing morphogenesis ${ }^{6,7}$. However, the mechanical role of the basement membrane in post-implantation embryogenesis remains unknown. Here we demonstrate the importance of spatiotemporally regulated basement membrane remodelling during early embryonic development. Specifically, we show that Nodal signalling directs the generation and dynamic distribution of perforations in the basement membrane by regulating the expression of matrix metalloproteinases. This basement membrane remodelling facilitates embryo growth before gastrulation. The establishment of the anterior-posterior axis ${ }^{8,9}$ further regulates basement membrane remodelling by localizing Nodal signalling-and therefore the activity of matrix metalloproteinases and basement membrane perforations-to the posterior side of the embryo. Perforations on the posterior side are essential for primitive-streak extension during gastrulation by rendering the basement membrane of the prospective primitive streak more prone to breaching. Thus spatiotemporally regulated basement membrane remodelling contributes to the coordination of embryo growth, morphogenesis and gastrulation.
The early post-implantation mouse embryo is a hollowed cylindrical structure, the 'egg cylinder', which consists of two abutting tissues, the pluripotent epiblast and the extraembryonic ectoderm, and is overlaid by a layer of another extraembryonic tissue, the visceral endoderm ${ }^{10}$. After implantation, the epiblast and extraembryonic ectoderm grow along the embryo's proximal-distal axis despite being surrounded by a laminin- and collagen-rich basement membrane ${ }^{11}$, which is important for maintaining the shape of the embryo (Extended Data Fig. 1a-c).

Of note, there is no evidence of basement membrane dissolution during embryo growth until gastrulation. As a nondeformable basement membrane would present a barrier to tissue expansion, we hypothesized that it must have special features to permit embryo growth. To address this hypothesis, we examined the development of mouse embryos from implantation until gastrulation (embryonic day (E) 5.0E6.5), revealing perforations in the basement membrane surrounding the epiblast (Fig. 1a, b, Extended Data Fig. 1d), the region that experiences higher forces owing to the direction of embryo growth. Most of these perforations $(99 \%, n=1,502$ perforations quantified) were smaller than the average size of cell nuclei, limiting epiblast-cell transmigration into the visceral endoderm, as reported to occur at the distal part of in vitro-cultured embryos ${ }^{12,13}$ (Extended Data Fig. 1e-g). Similar basement membrane perforations have been observed during branching morphogenesis of several embryonic mouse tissues ${ }^{5}$, but their presence during early post-implantation development was previously unknown.

Our analyses revealed that the basement membrane perforations were initially evenly distributed around the epiblast but becameskewed towards one side of the embryo as it grew (Fig.1a, b, $n=90$, Supplementary Video1). To assess whether the onset of this asymmetry might follow the establishment of the anterior-posterior (AP) axis, we examined the distribution of perforations relative to the position of the anterior visceral endoderm (AVE), which migrates towards one side of the embryo and initiates AP patterning by inhibiting Nodal and Wnt signalling in adjacent epiblast ${ }^{14}$ (Fig. 2a, Extended Data Fig. 2a). We found fewer basement membrane perforations in the AVE region and that basement membrane perforations were localized on the posterior side upon completion of AVE repositioning (Fig. 2a, b, Extended Data Fig. 2a, b, Supplementary Videos 2-4).

To test whether AP axis specification regulates the position of perforations, we examined the consequences of inhibiting AVE migration

${ }^{1}$ Mammalian Embryo and Stem Cell Group, Department of Physiology, Development and Neuroscience, University of Cambridge, Cambridge, UK. ' ${ }^{2}$ Department of Genetics, University of Cambridge, Cambridge, UK. ${ }^{3}$ Centre of Trophoblast Research, University of Cambridge, Cambridge, UK. ${ }^{4}$ IRIBHM, Université Libre de Bruxelles, Brussels, Belgium. ${ }^{5}$ Division of Biology and Biological Engineering, California Institute of Technology, Pasadena, CA, USA. ${ }^{6}$ Present address: Department of Biological Sciences, University of Cyprus, Nicosia, Cyprus. ${ }^{7}$ These authors contributed equally: Christos Kyprianou, Neophytos Christodoulou. ${ }_{\mathrm{e}-\mathrm{mail}}$ mz205@cam.ac.uk 


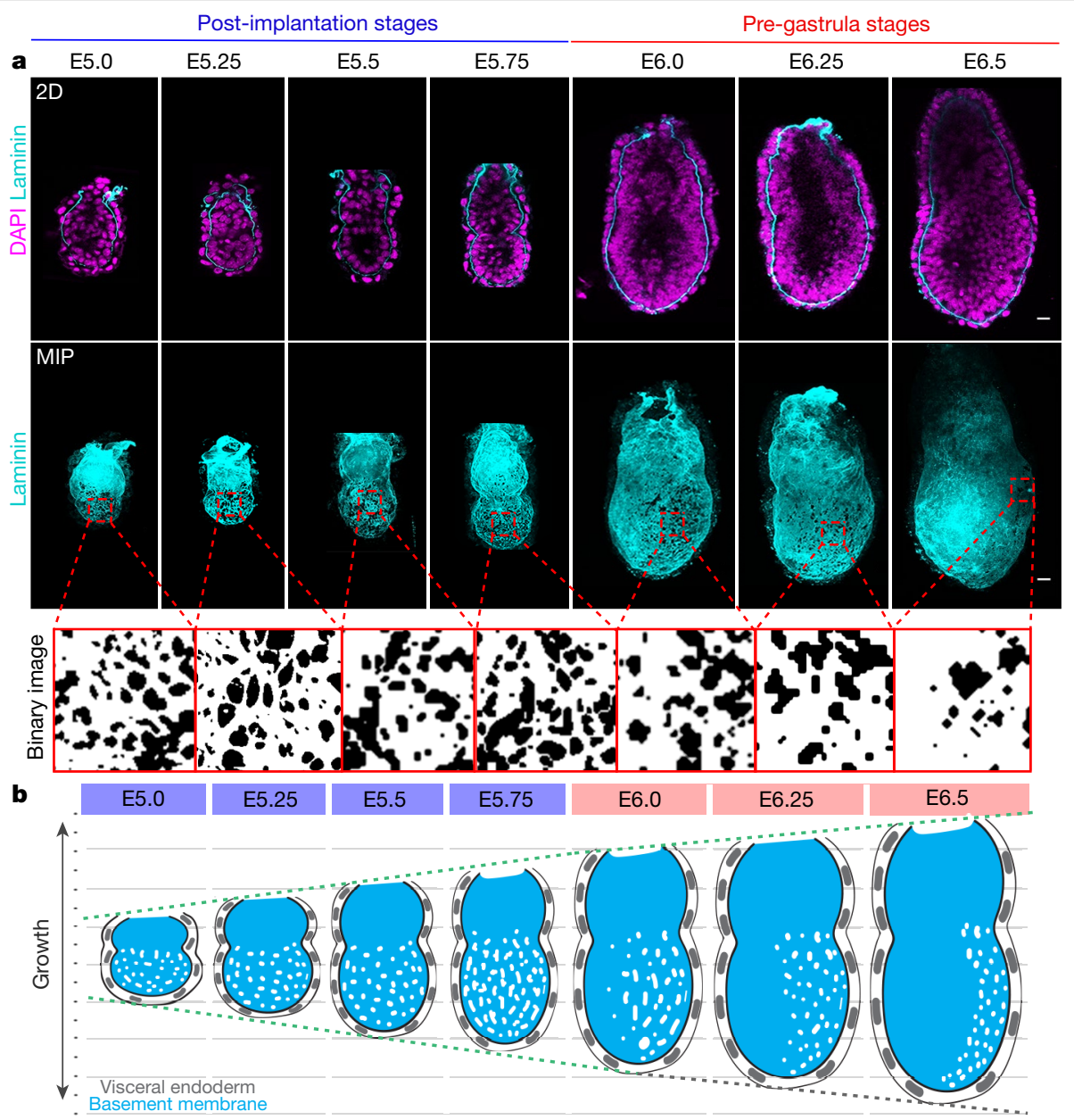

Fig. 1 | Basement membrane architecture during pre-gastrula stages. a, The early post-implantation embryos show a uniform distribution of perforations, which becomes progressively asymmetric in the pre-gastrula stages. Binary images (bottom) highlight apparent perforations on the basement membrane. $n=10$ embryos per stage. MIP, maximum intensity projection. $\mathbf{b}$, Schematic representation of basement membrane architecture. The grid and dotted lines show the relative increase in embryo size. Blue, post-implantation stages; red, pre-gastrula stages. Scale bars, $20 \mu \mathrm{m}$. by disrupting RhoA, which is required for collective cell migration ${ }^{15}$. To this end, we examined the development of visceral endoderm-specific Rhoa-knockout embryos (Ttr-cre; Rho $a^{f /-}$ ). As expected, the AVE migrated unilaterally in control embryos, but remained positioned distally in Ttr-cre;Rhoa ${ }^{f l-}$ embryos (Fig. 2c, Extended Data Fig. 2c). Notably, basement membrane perforations accumulated proximally and failed to localize asymmetrically along an axis perpendicular to the proximaldistalaxis of the Ttr-cre;Rho $a^{f /-}$ embryo (Fig. 2c-e,ExtendedDataFig.2c,d). These results indicate that the distribution of basement membrane perforations might be linked to AVE migration and AP axis establishment.

The AVE contributes to the establishment of a Nodal activity gradient across the AP axis ${ }^{8,9}$, which is instrumental for positioning the primitive streak and initiating gastrulation ${ }^{16}$. We hypothesized that the AVE-mediated regulation of Nodal signalling could control the distribution of basement membrane perforations. To test this hypothesis, we cultured tamoxifen-inducible Nodal-knockout E5.75 embryos $\left(\text { Nodal }^{l / f l} \mathrm{CreER}^{T 2}\right)^{17}$ in the presence of 4-hydroxytamoxifen for $18 \mathrm{~h}$. We found that Nodal-knockout embryos lacked basement membrane perforations and accumulated laminin around the epiblast, in contrast to control embryos (Fig. 3a, b, Extended Data Fig. 3a). Pharmacological inhibition of Nodal resulted in a similar phenotype (Extended Data Fig. 3b, c). These results indicate that Nodal activity is required for the basement membrane remodelling.

We next wished to determine whether the epiblast is sufficient to induce basement membrane remodelling. To this end, we used embryonic stem (ES) cells, which model the transcriptional profile of the early post-implantation epiblast ${ }^{18}$. Thus, we cultured ES cells using fluorescent gelatin (Cy3-gelatin) as an artificial substitute for the basement membrane, and induced pluripotency exit in the ES cells by removing the pluripotency maintenance factors LIF, PD0325901 and CHIR99021 (PD0325901 and CHIR99021 are MEK and GSK3 inhibitors, respectively; hereafter referred to as 2i) (Extended Data Fig. 3d-f). Under these conditions, ES cells induced perforations in the gelatin, whereas they failed to do so in the presence of 2i-LIF (Extended Data Fig. 3d, e). Thus, ES cells cultured in an early post-implantation-like state can remodel the underlying extracellular matrix (ECM). Treatment with the Nodal inhibitor SB431542 blocked the formation of perforations in the gelatin layer, confirming that Nodal signalling is required for basement membrane remodelling in vitro and in vivo (Extended Data Fig. 3f, g). These data suggest that ES cells can directly remodel the basement membrane in a Nodal-dependent manner upon pluripotency exit.

The matrix metalloproteinase (MMP) family of proteins promote basement membrane breakdown in various cell types. MMPs have key roles in enabling cancer metastasis ${ }^{19,20}$ and the developmental remodelling of basement membranes ${ }^{20}$. Because inhibition of MMPs impairs the formation of basement membrane perforations in mouse salivary glands ${ }^{5}$, we considered whether MMPs might be involved in generating basement membrane perforations during mouse embryogenesis. To test this, we analysed the expression of MMP family members during post-implantation development using a single-cell sequencing datase $t^{21}$ 


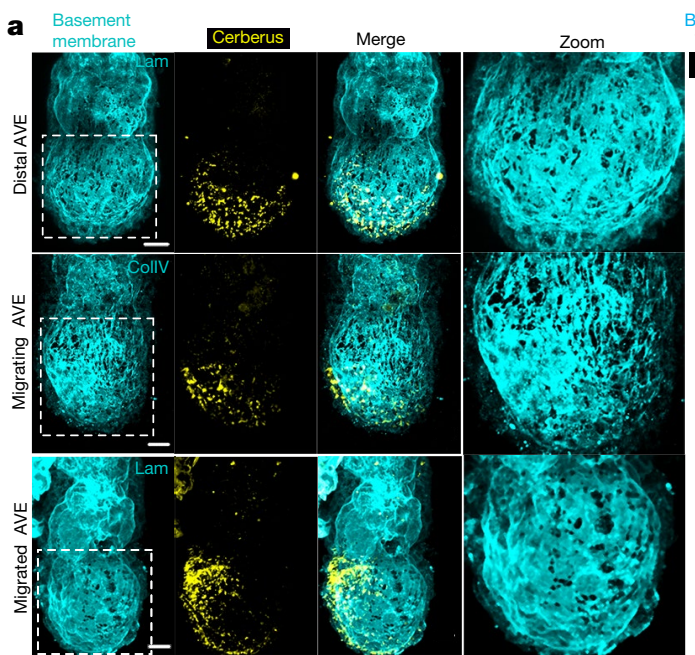

Basement membrane
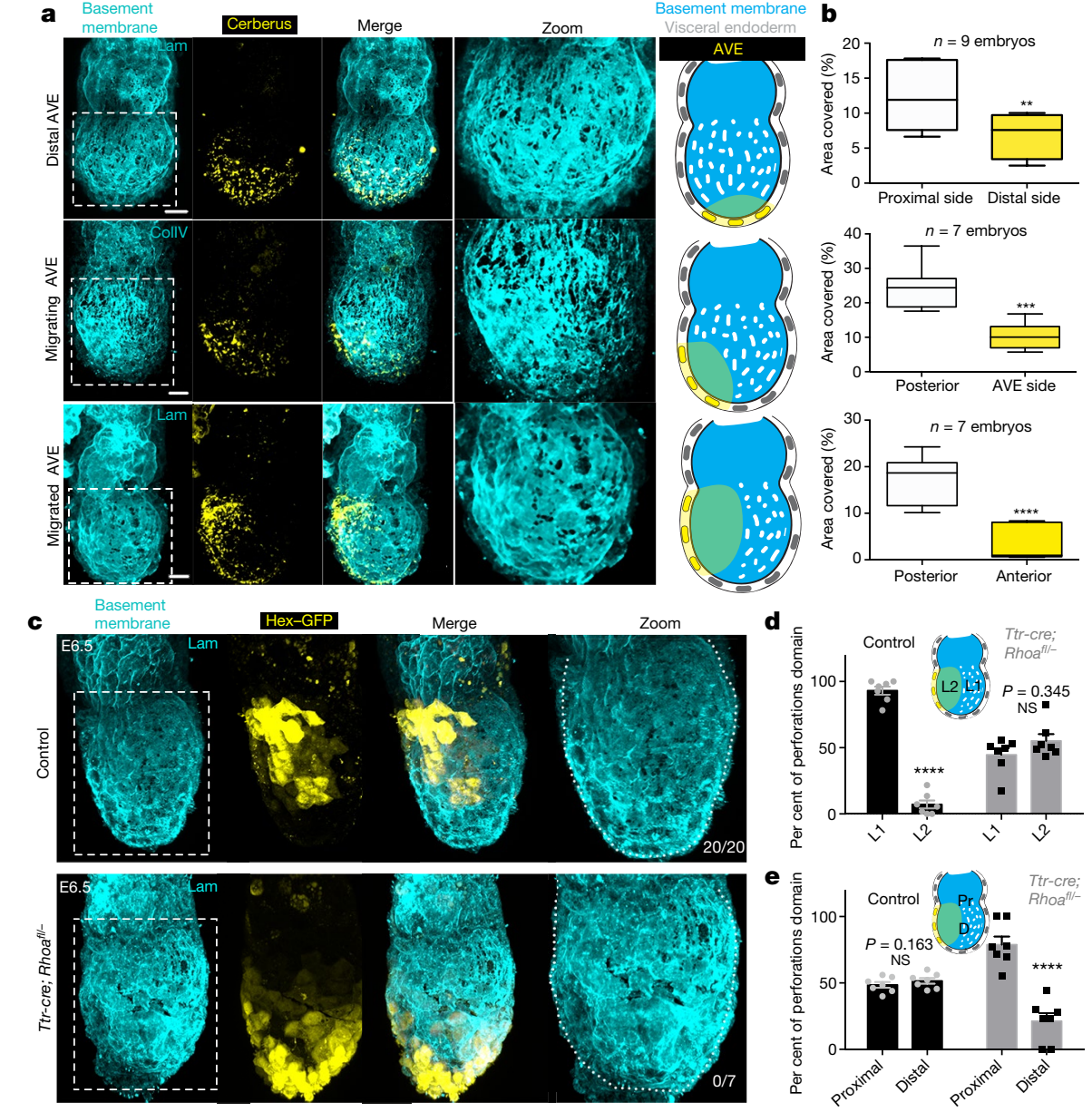

Fig. 2 | AVE positioning and basement membrane remodelling. a, Correlation of AVE migration (visualized using a Cerberus antibody) and basement membrane remodelling. $n=40$ embryos. Lam, laminin; ColIV, collagen IV.b, Quantification of basement membrane area covered by perforations at regions away from or close to the AVE population at different stages of AVE migration. Two-tailed unpaired Student's $t$-test; ${ }^{* *} P=0.0068$, ${ }^{* * *} P=0.0002,{ }^{* * * *} P<0.0001$. In box plots, centre lines show median values, box limits represent the upper and lower quartiles, and whiskers show the range of values. c, Representative examples of pre-gastrula control and Ttr-cre;Rhoa ${ }^{f l-}$ embryos. Inhibition of AVE (visualized by a Hhex-EGFP transgene, Hex-GFP) migration results in abnormal distribution of basement membrane perforations and their accumulation at the proximal side. $n=20$ control, $n=7$ Ttr-cre; Rhoa ${ }^{f l-}$ embryos. The fractions in the bottom right indicate the number of embryos with a migrated AVE out of all embryos recovered per group. Dotted white line outlines the embryonic basement membrane.d, e, Localization of the domains of perforation: lateral domain 1(L1) and lateral domain 2 (L2) (d), and proximal (Pr) and distal (D) domains (e). Two-tailed unpaired Student's $t$-test; ${ }^{* * * *} P<0.0001$; NS, not significant; mean \pm s.e.m. $n=7$ control, $n=7$ Ttr-cre; Rhoa ${ }^{f l-}$ embryos. Scale bars, $20 \mu \mathrm{m}$.
(Extended Data Fig. 4a, b). To select target genes, we set three criteria. First, the candidate MMP genes had to be expressed in the epiblast throughout post-implantation development. Second, MMP candidate genes had to show posterior localization of their expression in the pre-gastrula stages in a similar position to the basement membrane perforations. Finally, the MMP candidate-gene products had to be able to cleave both laminin and collagen. Our analysis revealed that three MMP genes (Mmp2, Mmp14 and Mmp25) are expressed in the epiblast of post-implantation embryos (Extended Data Fig. 4c). Of these candidate genes, only Mmp2 and Mmp14 are expressed in the posterior region in the pre-gastrula stage (Fig. 3c, d, Extended Data Fig. 4d-f). Furthermore, whereas MMP2 and MMP14 can cleave both laminin and collagens $^{22}$, MMP25 cleaves collagen but does not cleave laminin ${ }^{23}$. On the basis of these findings, we excluded $M m p 25$ as a candidate-gene product and focused on $M m p 2$ and Mmp14.

ECM components showed no preferential anterior expression in the epiblast or the visceral endoderm upon redistribution of perforations to the posterior side of the embryo ${ }^{21}$ (Extended Data Fig. 5a), suggesting that the expression of ECM components is not regulated by Nodal signalling. Thus, the loss of basement membrane perforations in Nodal-knockout embryos cannot be accounted for by increased expression of constituent ECM proteins, in agreement with previous results showing that the expression of ECM components surrounding the epiblast is not affected in Smad4-knockout embryoid bodies ${ }^{24}$. Given that Nodal signalling regulates basement membrane remodelling and that expression of Mmp2,Mmp14 and genes involved in Nodal signalling follows the same pattern, we hypothesized that Nodal regulates the expression of MMPs. To test this hypothesis, we examined the expression of MMP2 and MMP14 in control, SB431542-treated and Nodal-knockout embryos. Our analyses indicate that Nodal regulates expression of MMP2 and MMP14 during post-implantation development (Fig. 3e-h, Extended Data Fig. 5b, c). In accordance with these results, levels of MMP2 and MMP14 were also reduced in ES cells treated with Nodal inhibitor upon exit from pluripotency (Extended Data Fig. 5d, e). To examine whether Nodal regulates expression of Mmp2 and Mmp14 directly, we analysed datasets from chromatin immunoprecipitation with sequencing (ChIP-seq) of Smad $2 / 3^{25}$, generated using embryoid bodies treated with activin to activate Nodal or with SB431542 to inhibit Nodal. This analysis indicated that Smad2/3 binds to the promoters of Mmp2 and Mmp14 in a Nodal-dependent manner 


\section{Article}
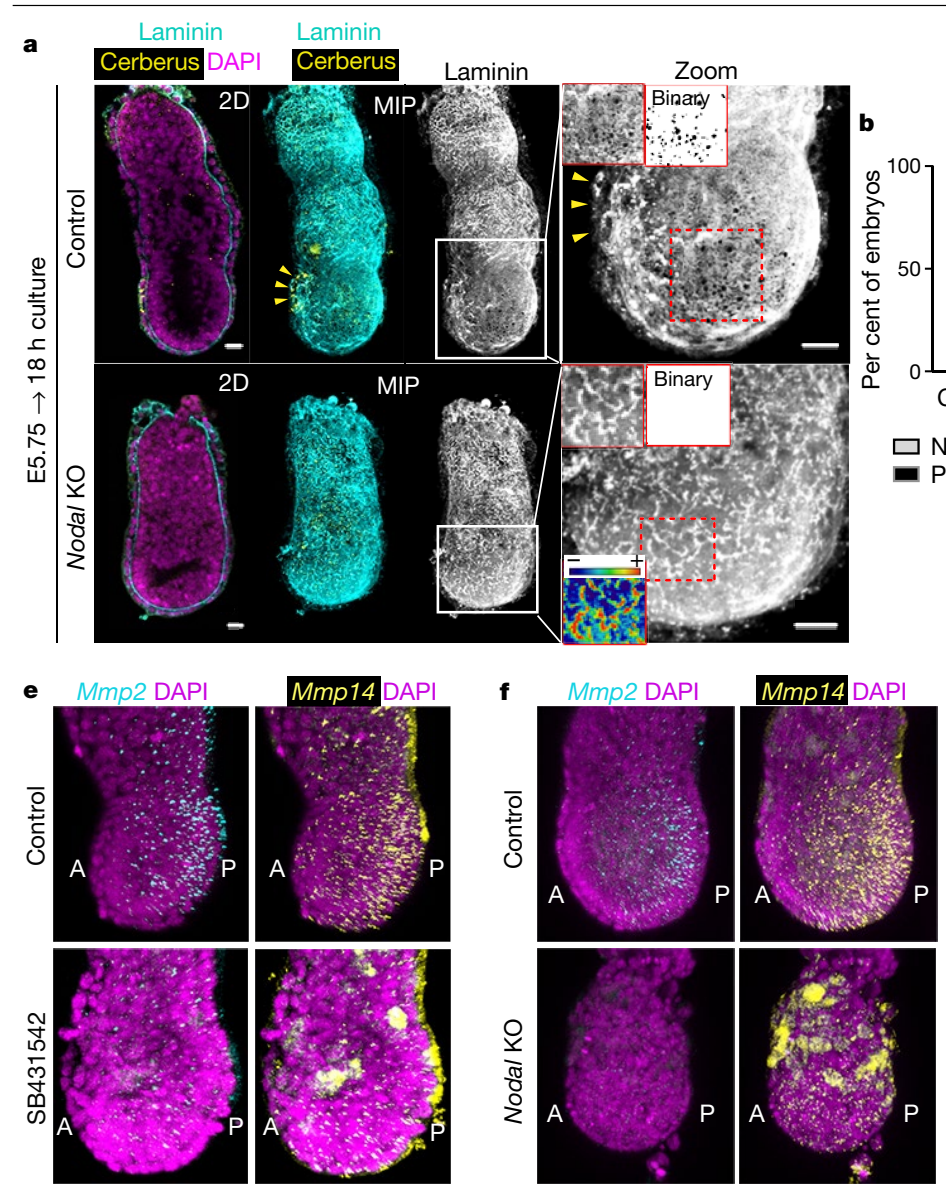

Fig. 3 | Nodal signalling regulates basement membrane remodelling via regulation of MMP expression. a, Control (Nodal $\left.l^{f l f l} \mathrm{CreER}^{T 2} ; n=10\right)$ and Nodal-knockout (KO) (Nodal ${ }^{l / f l}{ }^{\prime}$ creER $R^{T 2}+$ tamoxifen; $\left.n=17\right)$ embryos. Yellow arrowheads, AVE region. Top, insets show zoomed epiblast with binary image. Bottom, inset shows intensity-colour-coded image revealing the accumulation of basement membrane in Nodal-KO embryos. b, Quantification of basement membrane perforations in control and Nodal-KO embryos. $\mathrm{X}^{2}$ test;

${ }^{* * * *} P<0.0001$. c, Expression levels of Mmp2, Mmp14, Mmp25 and posterior marker genes ( $T$, Nodal and $T d g f 1)$ are plotted on the uniform manifold approximation and projection (UMAP) for E6.25 and E6.5 epiblast cells ${ }^{21}$ ( $n=527$ cells). Expression is expressed as $\log$ (counts). Green arrowheads,

(Extended Data Fig. 5f). In agreement with these results, MMP14 was shown to be downregulated in Smad4-knockout embryoid bodies ${ }^{24}$. Our results indicate that Nodal signalling directly regulates the expression of MMP2 and MMP14 through Smad2/3 during early post-implantation development, although it is possible that independent mechanisms regulated by Nodal signalling can also contribute to the regulation of MMP expression.

If Nodal drives basement membrane remodelling by promoting MMP expression, we would expect MMP activity to be required for remodelling the underlying ECM. To determine whether this is the case, we gen-

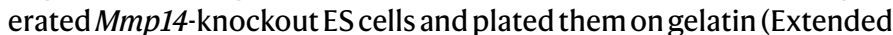
Data Fig. 6a-d). We found that, upon exit from pluripotency, the Mmp14-knockout ES cells failed to generate perforations in the gelatin, unlike the wild-type control cells (Extended Data Fig. 6e,f). As MMP14 is also an activator of $\mathrm{MMP}^{19}$, these results indicate that knockout of two major MMPs is sufficient to block perforation in vitro. However, Mmp14-single knockout and Mmp2-Mmp14 double-knockout embryos do not show early developmental phenotypes in vivo ${ }^{26,27}$. Because enzymatic redundancy among MMP family members can mask developmental phenotypes ${ }^{26,27}$, we investigated the role of MMPs in basement membrane remodelling by inhibiting MMPs pharmacologically.

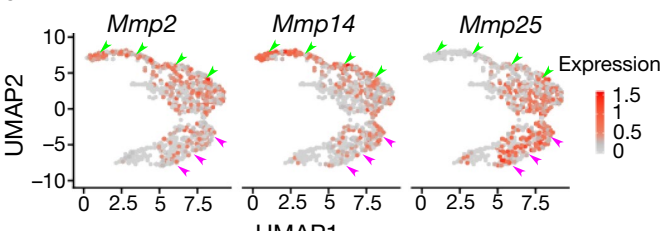

UMAP1

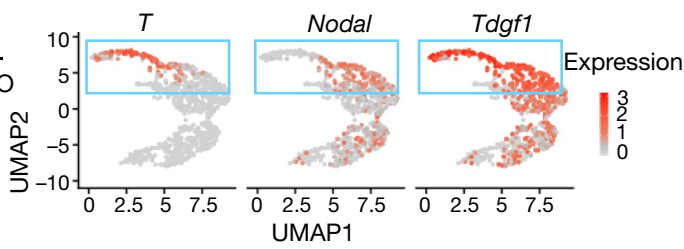

d

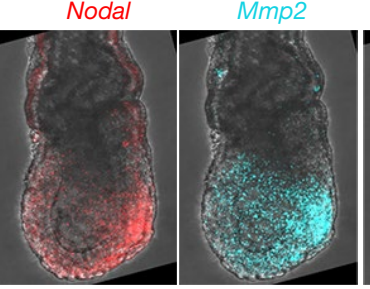

Mmp14

Merge
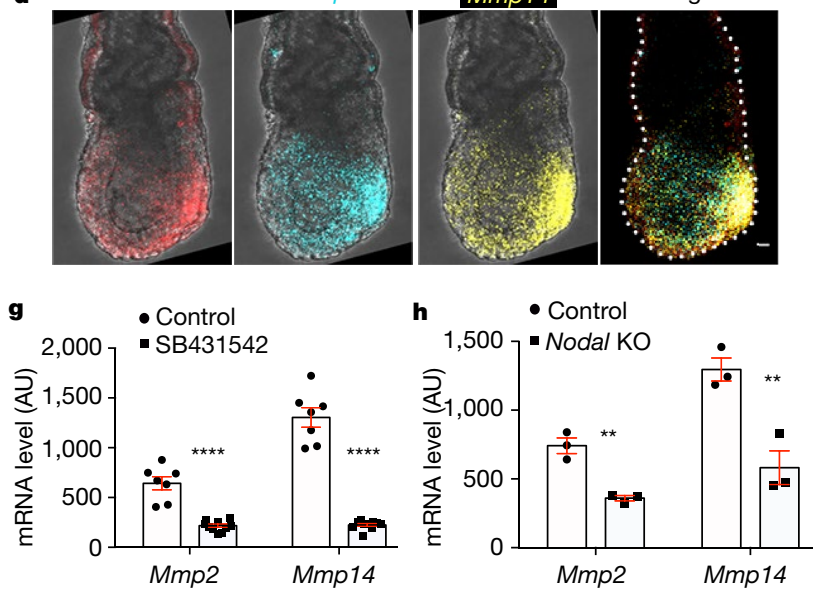

posterior epiblast; purple arrowheads, anterior epiblast; blue box, posterior epiblast. d, Whole-mount fluorescent RNA in situ hybridization (FISH) of a representative $\mathrm{E} 6.5$ embryo superimposed on the bright-field image. $n=5$ embryos. e,f, Representative examples of control, Nodal inhibitor-treated (e) and Nodal-KO (f) embryos analysed by whole-mount FISH for Mmp2 and Mmp14 expression. A, anterior; $\mathrm{P}$, posterior. $\mathbf{g}, \mathbf{h}$, Quantification of $\mathrm{Mmp2}$ and $\mathrm{Mmp14}$ mRNA in control, $18 \mathrm{~h}$ Nodal inhibitor-treated (g) and Nodal-KO (h) embryos. Two-sided unpaired Student's $t$-test; ${ }^{* * * *} P<0.0001,{ }^{* *} P=0.0031(\mathrm{Mmp})$; ${ }^{* *} P=0.0086$ (Mmp14); mean \pm s.e.m. In e, $\mathbf{g}, n=7$ (control) and 10 (Nodal inhibitor-treated) embryos. In $\mathbf{f}, \mathbf{h}, n=3$ (control) and 3 (Nodal-KO) embryos. Scale bars, $20 \mu \mathrm{m}$. AU, arbitrary units.

WeculturedE5.75 embryos for $18 \mathrm{~h}$ in the presence of the broad-spectrum MMP inhibitor prinomastat ${ }^{28}$ complemented with the MMP14-specific inhibitor NSC405020, which inhibits the MMP14 homodimerization necessary for its collagenolytic function ${ }^{29}$. Embryos treated with MMP inhibitors lacked basement membrane perforations and phenocopied the basement membrane architecture of Nodal-knockout embryos (Fig. 4a, b).

As our results indicated that MMP inhibition also led to reduced growth and increased apoptosis in the epiblast (Fig. 4c, Extended Data Fig. 7a-g, Supplementary Videos 5, 6), we also examined ES cell growth in 3D, which recapitulates the growth of the post-implantation epiblast $^{30}$. We found that broad inhibition of MMP activity or genetic loss of $\mathrm{Mmp14}$ led to significantly reduced growth of ES cell spheroids and increased apoptosis (Extended Data Fig. 8a-e). To evaluate the possibility that the inhibitor treatment is toxic, we treated ES cells and extraembryonic endoderm stem cells (XEN cells) in 2D cultures with the same concentration of inhibitors. We found that MMP inhibition had no effect on cell survival (Extended Data Fig. 8f), suggesting that phenotypes observed in embryos and ES cells cultured in 3D are specific to growth restriction. In support of this conclusion, we could partially rescue the growth deficiency of ES cell spheroids treated with MMP 

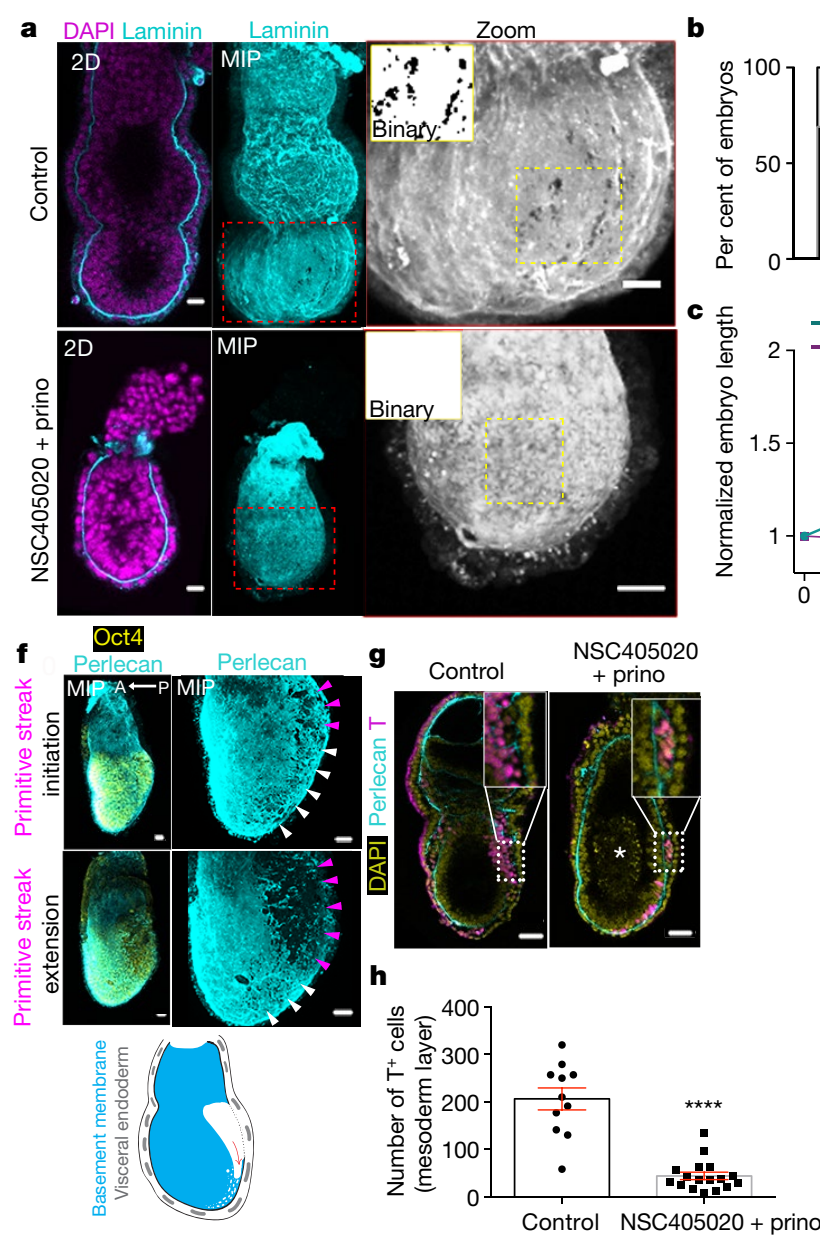

Fig. 4 | MMP-mediated basement membrane remodelling permits growth and primitive-streak extension. a, Laminin immunofluorescence in control $(n=13)$ and MMP inhibitor-treated $(n=16)$ embryos. Prino, prinomastat.

b, Quantification of basement membrane perforations. $X^{2}$ test; ${ }^{* * * *} P<0.0001$. c, Quantification of embryo length over time. Mean \pm s.e.m. d, Rose diagrams for vector angles in E5.0-E5.25 (before growth) and E5.5-E5.75 (during growth) embryos. E5.0-E5.25: $n=10$ embryos, 1,288 vectors; E5.5-E5.75: $n=10$ embryos, 1,481 vectors. Kolmogorov-Smirnov test; ${ }^{* * *} P<0.0001$. e, Model for the correlation between basement membrane perforations and embryo growth.f, Top, Perlecan immunofluorescence in representative E6.5 embryos.
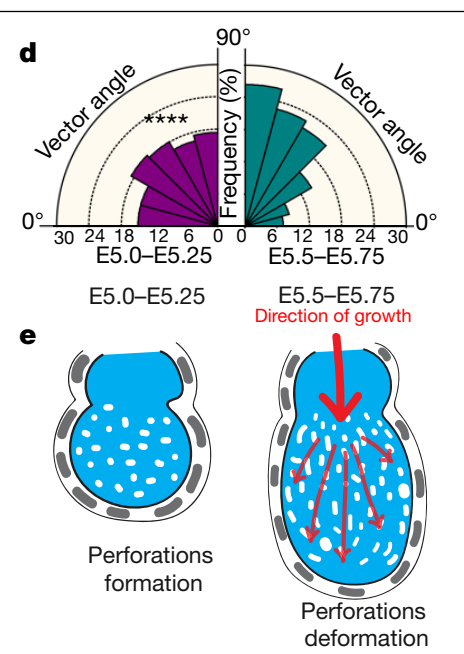
Time ( $h$ )

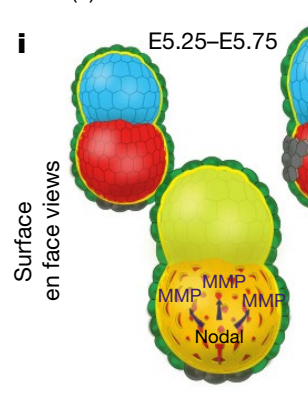

Embryo growth
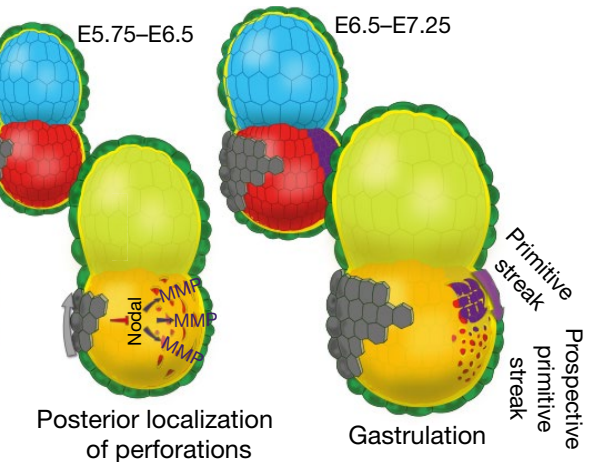
of perforations

Basement membrane
Visceral endoderm
AVE

Extraembryonic ectoderm
Epiblast
Mesoderm

White arrowheads, prospective primitive streak. Purple arrowheads: primitive streak and breached basement membrane. $n=30$ embryos. Bottom, schematic showing the 'run in the stocking' model for primitive-streak extension along the perforation domain. $g$, Immunofluorescence of representative examples of embryos cultured for $18 \mathrm{~h}$ from early E6.5 (pre-primitive-streak stage). Scale bars, $50 \mu \mathrm{m}$. Asterisk, dead cells. h, Progression of gastrulation. Two-sided unpaired Student's $t$-test; ${ }^{* * * *} P<0.0001$; mean \pm s.e.m. In $\mathbf{g}, \mathbf{h}, n=11$ (control) and $n=17$ (MMP inhibitor-treated) embryos. $\mathbf{i}$, Model for regulation of basement membrane remodelling up to gastrulation. In a, f, scale bars, $20 \mu \mathrm{m}$.

Data Fig. 9a-d, Supplementary Videos 2-4). The presence of perforations at the posterior from $12 \mathrm{~h}$ before the initiation of gastrulation (Extended Data Fig. 9c) suggests that basement membrane perforations might enable the formation of the primitive streak. Consistent with this possibility, we observed that proximo-posterior breaching of the basement membrane, denoting the initiation of gastrulation, aligned with the perforations (Fig. 4f, Extended Data Fig. 10a, b, Supplementary Video 7). On the basis of these observations, we hypothesized that the perforations at the position of the prospective primitive streak might render the basement membrane more prone to breakage to facilitate gastrulation (Fig. $4 \mathrm{f}$, bottom). This can be explained by the 'run in the stocking model', which proposes that localized remodelling of the basement membrane leads to its weakening at a specific domain, which will open and expand upon exposure to tension induced by tissue growth $^{32}$. To test whether this model could apply to primitive-streak progression, we cultured E6.5 embryos, adding MMP inhibitors just before initiation of the primitive streak. We found that inhibition of MMPs led to incomplete mesoendoderm (T-expressing cells) ingression, defective primitive-streak extension and consequently, failure of gastrulation. This failure of the primitive streak to extend resulted in 


\section{Article}

the accumulation of mesoendoderm cells in the epiblast, the presence of dead cells in the pro-amniotic cavity, and the lack of extraembryonic mesoderm, anterior primitive streak and any laterally migrating mesoderm (Fig. 4g, h, Extended Data Fig. 10c-e, Supplementary Videos 8-11). These results suggest that the perforations in the posterior domain of the basement membrane are important to enable primitive-streak extension and normal gastrulation.

In conclusion, we have uncovered a mechanism linking the remodelling of the basement membrane with Nodal signalling and the establishment of the AP axis (Fig. 4i). We show that at early post-implantation stages, basement membrane perforations are evenly distributed, accommodating embryo growth. These perforations are produced through the expression of MMPs. As the AVE migrates to the future anterior, Nodal activity becomes restricted to the opposite side, enriching MMP expression-and thus perforations-in the future posterior. Finally, the perforations persist after initiation of gastrulation to line the prospective primitive streak. This pre-patterning of the basement membrane at the site of the primitive streak is important for correct progression of gastrulation and primitive-streak extension. Thus, remodelling of the basement membrane coordinates growth and morphogenesis in the embryo to enable proper gastrulation.

\section{Online content}

Any methods, additional references, Nature Research reporting summaries, source data, extended data, supplementary information, acknowledgements, peer review information; details of author contributions and competing interests; and statements of data and code availability are available at https://doi.org/10.1038/s41586-020-2264-2.

1. Keller, R. Shaping the vertebrate body plan by polarized embryonic cell movements. Science 298, 1950-1954 (2002).

2. Martin, A. C. \& Goldstein, B. Apical constriction: themes and variations on a cellular mechanism driving morphogenesis. Development 141, 1987-1998 (2014).

3. Crest, J., Diz-Muñoz, A., Chen, D. Y., Fletcher, D. A. \& Bilder, D. Organ sculpting by patterned extracellular matrix stiffness. elife 6, e24958 (2017).

4. Haigo, S. L. \& Bilder, D. Global tissue revolutions in a morphogenetic movement controlling elongation. Science 331, 1071-1074 (2011).

5. Harunaga, J. S., Doyle, A. D. \& Yamada, K. M. Local and global dynamics of the basement membrane during branching morphogenesis require protease activity and actomyosin contractility. Dev. Biol. 394, 197-205 (2014).

6. Bedzhov, I. \& Zernicka-Goetz, M. Self-organizing properties of mouse pluripotent cells initiate morphogenesis upon implantation. Cell 156, 1032-1044 (2014).

7. Christodoulou, N. et al. Sequential formation and resolution of multiple rosettes drive embryo remodelling after implantation. Nat. Cell Biol. 20, 1278-1289 (2018).

8. Yamamoto, M. et al. Nodal antagonists regulate formation of the anteroposterior axis of the mouse embryo. Nature 428, 387-392 (2004).
9. Brennan, J. et al. Nodal signalling in the epiblast patterns the early mouse embryo. Nature 411, 965-969 (2001)

10. Christodoulou, N. et al. Morphogenesis of extra-embryonic tissues directs the remodelling of the mouse embryo at implantation. Nat. Commun. 10, 3557 (2019).

11. Copp, A. J. Interaction between inner cell mass and trophectoderm of the mouse blastocyst. I. A study of cellular proliferation. J. Embryol. Exp. Morphol. 48, 109-125 (1978).

12. Hiramatsu, R. et al. External mechanical cues trigger the establishment of the anteriorposterior axis in early mouse embryos. Dev. Cell 27, 131-144 (2013).

13. Bedzhov, I. et al. Development of the anterior-posterior axis is a self-organizing process in the absence of maternal cues in the mouse embryo. Cell Res. 25, 1368-1371 (2015).

14. Thomas, P. Q., Brown, A. \& Beddington, R. S. Hex: a homeobox gene revealing peri-implantation asymmetry in the mouse embryo and an early transient marker of endothelial cell precursors. Development 125, 85-94 (1998).

15. Reffay, M. et al. Interplay of RhoA and mechanical forces in collective cell migration driven by leader cells. Nat. Cell Biol. 16, 217-223 (2014).

16. Conlon, F. L. et al. A primary requirement for nodal in the formation and maintenance of the primitive streak in the mouse. Development 120, 1919-1928 (1994).

17. Kumar, A., Lualdi, M., Lewandoski, M. \& Kuehn, M. R. Broad mesodermal and endoderma deletion of Nodal at postgastrulation stages results solely in left/right axial defects. Dev. Dyn. 237, 3591-3601 (2008)

18. Kalkan, T. et al. Tracking the embryonic stem cell transition from ground state pluripotency. Development 144, 1221-1234 (2017).

19. Lemaître, V. \& D'Armiento, J. Matrix metalloproteinases in development and disease. Birth Defects Res. C 78, 1-10 (2006)

20. Lu, P., Takai, K., Weaver, V. M. \& Werb, Z. Extracellular matrix degradation and remodeling in development and disease. Cold Spring Harb. Perspect. Biol. 3, a005058 (2011).

21. Cheng, S. et al. Single-cell RNA-seq reveals cellular heterogeneity of pluripotency transition and X chromosome dynamics during early mouse development. Cell Rep. $\mathbf{2 6}$ 2593-2607 (2019).

22. Klein, T. \& Bischoff, R. Physiology and pathophysiology of matrix metalloproteases. Amino Acids 41, 271-290 (2011).

23. English, W. R., Velasco, G., Stracke, J. O., Knäuper, V. \& Murphy, G. Catalytic activities of membrane-type 6 matrix metalloproteinase (MMP25). FEBS Lett. 491, 137-142 (2001).

24. Costello, I., Biondi, C. A., Taylor, J. M., Bikoff, E. K. \& Robertson, E. J. Smad4-dependent pathways control basement membrane deposition and endodermal cell migration at early stages of mouse development. BMC Dev. Biol. 9, 54 (2009).

25. Wang, Q. et al. The p53 family coordinates Wnt and Nodal inputs in mesendodermal differentiation of embryonic stem cells. Cell Stem Cell 20, 70-86 (2017)

26. Holmbeck, K. et al. MT1-MMP-deficient mice develop dwarfism, osteopenia, arthritis, and connective tissue disease due to inadequate collagen turnover. Cell 99, 81-92 (1999).

27. Oh, J. et al. Mutations in two matrix metalloproteinase genes, MMP-2 and MT1-MMP, are synthetic lethal in mice. Oncogene 23, 5041-5048 (2004).

28. Coussens, L. M., Fingleton, B. \& Matrisian, L. M. Matrix metalloproteinase inhibitors and cancer: trials and tribulations. Science 295, 2387-2392 (2002)

29. Remacle, A. G. et al. Novel MT1-MMP small-molecule inhibitors based on insights into hemopexin domain function in tumor growth. Cancer Res. 72, 2339-2349 (2012).

30. Shahbazi, M. N. et al. Pluripotent state transitions coordinate morphogenesis in mouse and human embryos. Nature 552, 239-243 (2017).

31. Ben-Haim, N. et al. The Nodal precursor acting via activin receptors induces mesoderm by maintaining a source of its convertases and BMP4. Dev. Cell 11, 313-323 (2006).

32. Moore, K. A. et al. Control of basement membrane remodeling and epithelial branching morphogenesis in embryonic lung by Rho and cytoskeletal tension. Dev. Dyn. 232 268-281 (2005).

Publisher's note Springer Nature remains neutral with regard to jurisdictional claims in published maps and institutional affiliations.

(c) The Author(s), under exclusive licence to Springer Nature Limited 2020 


\section{Methods}

No statistical methods were used to predetermine sample size. The experiments were not randomized. The investigators were not blinded to allocation during experiments and outcome assessment.

\section{Embryo recovery and culture}

Mice were maintained in an animal facility in accordance with national and international guidelines. All experiments performed have been regulated by the Animals (Scientific Procedures) Act 1986 Amendment Regulations 2012 and additional ethical review by the University of Cambridge Animal Welfare and Ethical Review Body (AWERB). Experiments were authorised by the Home Office (licence number:70/8864). Upon any identification of health concern, mice were culled by cervical dislocation.

Embryos were recovered from wild type MF1 and CD1 female mice mated with MF1 males, dissected out of deciduae and transferred in drops of culture medium (Advanced DMEM/F12 (Thermo Fisher Scientific, 12634-010) with 30\% FBS (Stem Cell Institute) and supplemented with $2 \mathrm{mML}$-glutamine (Life Technologies, 35050-038), $1 \mathrm{mM}$ sodium pyruvate (Life Technologies, 11360039$)$, penicillin $\left(25 \mathrm{U} \mathrm{ml}^{-1}\right) /$ streptomycin $\left(25 \mathrm{~g} \mathrm{ml}^{-1}\right)$ (Thermo Fisher Scientific, 15140122), 1× insulin-transferrin-selenium-ethanolamine (ITS-X; Thermo Fisher Scientific, 51500056) for overnight culture or directly fixed in 4\% PFA (Electron Microscopy Sciences, 11586711; diluted in $1 \times$ phosphate buffered saline; PBS) for 20 min at room temperature or $100 \%$ methanol for $15 \mathrm{~min}$ at $-20^{\circ} \mathrm{C}$ (for MMP14 staining). Embryos cultured for imaging, were transferred to drops of medium on a glass-bottom dish (MatTek, P35G1.5-14-C) covered with mineral oil (Biocare, 9305). For MMP-inhibited embryo culture a combination of MMP inhibitors NSC405020 (Tocris, 4902;100 $\mu \mathrm{M}$ ) and prinomastat hydrochloride (Sigma-Aldrich, PZ0198; $20 \mu \mathrm{M})$ was used overnight $(18 \mathrm{~h})$. For visualizing cell death in MMP inhibitor-treated embryos in culture during live imaging, the cleaved-caspase live dye CellEvent Caspase-3/7 Green Detection Reagent (Thermo, C10723) was used at a concentration of $1 \mu \mathrm{M}$.

For the enzymatic removal of the basement membrane, embryos were cultured in the presence of collagenase IV $\left(500 \mu \mathrm{g} \mathrm{ml}^{-1}\right)$ for $3 \mathrm{~h}$ and then transferred in cultured medium supplemented with $50 \mu \mathrm{g} \mathrm{ml}^{-1}$ collagenase IV $15 \mathrm{~h}$.

To induce deletion of Nodal in the Nodaf ${ }^{f l / f l} \mathrm{CreER}^{T 2}$ recovered embryos were cultured in $2 \mu \mathrm{M}$ of (Z)-4-hydroxytamoxifen (in ethanol; Abcam, ab141943). Genotyping of single embryos following conversion of Nodat $t^{f l f l} \mathrm{CreER}^{T 2}$ was done using primers for detecting cre and primers for the wild-type and floxed alleles: cre forward, 5'-GCAGAACCTGAAGATGTTCGC-3'; cre reverse, 5'-AGGTATCTCTGACCAGAGTCA-3'; wild-type and floxed allele forward, 5'-ATGATTGTGGAGGAGTGTGGGTGC-3'; wild-type and floxed allele reverse: 5'-TCTCTGGCTTGGCAGGTCTAAG-3'.

PCR of knockout embryo genomic DNA showed a cre band and homozygous floxed allele. Embryos were cultured at $37^{\circ} \mathrm{C}$ in $5 \% \mathrm{CO}_{2}$.

\section{Visceral endoderm-specific $R$ hoa knockouts}

Female $R h o a^{f l f l}$ mice were crossed with $T$ tr-cre;Rhoa ${ }^{+/-}$males. Single-embryo genotyping for the Ttr-cre;Rhoa ${ }^{f l-}$ offspring was performed using one set of primers to check for presence of cre and another for detecting wild-type and floxed alleles: cre forward, 5'-GCAGAACCTGAAGATGTTCGC-3'; cre reverse, 5'-AGGTATCTCTGACCAGAGTCA-3'; wild-type and floxed allele forward, 5'-GACCAGCCTCTTGACCGATTTA-3', wild-type and floxed allele reverse primer, 5'-TGTGGGATACCGTTTGAGCAT-3'.

PCR of knockout embryo genomic DNA showed a cre band and homozygous floxed allele. Embryos were cultured at $37^{\circ} \mathrm{C}$ in $5 \% \mathrm{CO}_{2}$.

\section{Immunostaining}

Embryos fixed in 4\% PFA were first permeabilized for $30 \mathrm{~min}$ in $0.3 \%$ Triton X-100 and $0.1 \mathrm{M}$ glycine in PBS at room temperature. Methanol-fixed sample was not permeabilized further. The primary antibodies were added to the permeabilized embryos (washed three times in PBS) in blocking buffer $\left(0.1 \%\right.$ Tween-20, $10 \%$ filtered FCS in PBS) at $4{ }^{\circ} \mathrm{C}$ overnight. The primary antibodies were washed out by washing the embryos three times in PBS before adding the secondary antibodies in blocking buffer for 3-4 h at room temperature.

Primary antibodies: E-cadherin (1:300; Thermofisher Scientific, 13-1900), Oct4 (1:400; Santa Cruz, sc-5279), TFAP2C (1:200; Santa Cruz, sc-8977), laminin (1:400; Sigma, L9393), collagen IV (1:100; Millipore, AB769), HSPG2 (1:100; Millipore, MAB1948P), OTX2 (1:100; R\&D Systems, AF1979), Cerberus (1:500; R\&DSystems, MAB1986), Foxa2 (1:200; Cell Signalling Technologies, D56D6), T (also known as Brachyury) (R\&D Systems, MAB1556), cleaved caspase-3 (1:200; Cell Signalling Technologies, 9664S), MMP14 (1:150; Abcam, ab51074).

Secondary antibodies: Alexa Fluor 488 donkey anti-mouse (1:500; Thermo Fisher Scientific, A21202), Alexa Fluor 594 donkey anti-rat (1:500; Thermo Fisher Scientific, A21209), Alexa Fluor 647 donkey anti-rabbit (1:500; Thermo Fisher Scientific, A31573), Alexa Fluor 488 donkey anti-goat (1:500; Thermo Fisher Scientific, A11055).

F-actin staining: phalloidin 488 (1:500; Thermo Fisher Scientific, A12379). Nuclear staining: minimum 30 min incubation in DAPI(Thermo Fisher Scientific, D3571) + PBS $\left(5 \mathrm{mg} \mathrm{ml}^{-1}\right)$.

\section{Cell culture}

E14 and LifeAct-GFP ES cells (MMP14-KO or wild type) were used for this study. ES cells were cultured on $1 \%$ gelatin-coated dishes (Sigma-Aldrich, G2500) in the presence of $2 \mathrm{i}(3 \mu \mathrm{M}$ GSK3 inhibitor CHIR99021 (Stem Cell Institute), $1 \mu$ M MEK inhibitor PD0325901 (Stem Cell Institute)) and $10 \mathrm{ng} \mathrm{ml}^{-1}$ mouse LIF (Biochemistry Department, University of Cambridge). E14 base medium used was N2B27 (1:1 v/v DMEM/F12 (Thermo Fisher Scientific, 12634-010) and Neurobasal A (without vitamin A; Life Technologies, 10888-022), 1\% v/v B27 supplement (Thermo Fisher Scientific, A1895601), 0.5\% v/v N2 (DMEM/F12 (Thermo Fisher Scientific, 12634-010), $2.5 \mathrm{mg} \mathrm{ml}^{-1}$ insulin(Sigma-Aldrich, 19287), $10 \mathrm{mg} \mathrm{ml}^{-1}$ apo-transferrin (Sigma-Aldrich, T1147), 0.75\% v/v BSA fraction V (Thermo Fisher Scientific, 15260037), $20 \mathrm{\mu g} \mathrm{ml}^{-1}$ progesterone (Sigma-Aldrich, p8783), $1.6 \mathrm{mg} \mathrm{ml}^{-1}$ putrescine dihydrochloride (Sigma-Aldrich, P5780), $6 \mathrm{\mu g} \mathrm{ml}^{-1}$ sodium selenite (Sigma-Aldrich, S5261), $100 \mu \mathrm{M} \beta$-mercaptoethanol (Life Technologies, 31350-010), penicillin/streptomycin (Thermo Fisher Scientific, 15140122), GlutaMAX (Life Technologies, 35050-038)). Base medium for LifeAct-GFP cell culture was feeder cell (FC) medium (DMEM (Life Technologies, 41966052), 15\% FBS (Stem Cell Institute), penicillin/streptomycin (Thermo Fisher Scientific, 15140122), GlutaMAX (Life Technologies, 35050-038), nonessential aminoacids (Life Technologies, 11140035), sodium pyruvate (Life Technologies, 11360039), $100 \mathrm{U} \mu \mathrm{M}^{-1} \beta$-mercaptoethanol (Life Technologies, 31350-010)). Cells were passaged by detaching them with $0.05 \%$ Trypsin-EDTA (Life Technologies, 25300054) for 3 min at $37^{\circ} \mathrm{C}$. The trypsinization reaction was neutralized with $4 \times$ volume addition of FC medium and cells were collected and spun down at $1,000 \mathrm{~g}$ for $5 \mathrm{~min}$. Supernatant was then discarded, and cell pellet was resuspended in base medium and $2 i+$ LIF. Cells were then split 1:10 or 1:20 depending on the cell density. Cells were maintained in conditions to preserve stem cell character and prevent differentiation. The self-renewal properties of wild-type ES cells were confirmed routinely by immunofluorescence and morphological characteristics. Wild-type ES cell colonies maintained a domed morphology and XEN cells showed a mixed morphology of fibroblast-like and rounded cells as expected. All cell lines used in this study were routinely tested for mycoplasma contamination by PCR, which confirmed that they were negative for mycoplasma contamination.

\section{ES cell-spheroid generation}

E14 or LifeAct-GFP cells (Mmp14 knockout or wild type) were trypsinized with $0.05 \%$ Trypsin-EDTA (Life Technologies, 25300054) for 
3 min at $37^{\circ} \mathrm{C}$ when they reached $\sim 80 \%$ confluency. Cells were spun at $1,000 \mathrm{~g}$ for $5 \mathrm{~min}$ and supernatant discarded. The cell pellet was then resuspended in $1 \mathrm{ml}$ of base medium and were counted using a haemocytometer. The volume of cells taken for each experiment was calculated for 3,500-5,000 cells per $20 \mu \mathrm{l}$ drop of Matrigel (Corning, 354230 ). The appropriate volume was spun down again at $1,000 \mathrm{~g}$ for $5 \mathrm{~min}$ and supernatant removed. The cell pellet was resuspended in appropriate Matrigel concentration at a volume sufficient to plate $20 \mu \mathrm{l}$ drops of Matrigel on ibiTreat 8-well Ibidi plates (Ibidi, 80826). The suspended cells in Matrigel were placed at $37^{\circ} \mathrm{C}$ for 2 min to allow Matrigel polymerization. The drops were then covered with $250 \mu$ l of appropriate medium.

\section{Western blot}

Protein lysates were recovered from cells in 2D cultures using RIPA Buffer (1 M Tris-HCL, $1 \mathrm{M} \mathrm{NaCl}, 0.5 \mathrm{M}$ EDTA, 10\% TX-100, 10\% sodium deoxycholate, $10 \%$ SDS) with protease inhibitor cocktail (cOmplete Mini; Roche, 11836153001) on ice. After spinning at $16,900 \mathrm{~g}$ for $30 \mathrm{~min}$ at $4{ }^{\circ} \mathrm{C}$ the supernatant was collected and quantified for protein using a BCA assay kit (Pierce BCA Protein Assay Kit, 23227). Twenty micrograms of the sample was boiled at $96^{\circ} \mathrm{C}$ for $12 \mathrm{~min}$ in Laemlli buffer before being loaded in an $8 \%$ SDS-PAGE gel (stacking gel: $5 \%$ acrylamide mix (Severn Biotech, 20-210010), 120 mM Tris-pH 6.8, 0.1\% SDS (BioPath, S1030), 0.1\% ammonium persulfate (Sigma-Aldrich, 215589), 0.02\% v/v TEMED (Thermo Fisher Scientific, 17919); $8 \%$ resolving gel: $8 \%$ acrylamide mix, 375 mM Tris-pH 8.8, 0.1\% SDS, $0.1 \%$ ammonium persulfate, $0.06 \% \mathrm{v} / \mathrm{v}$ TEMED). After running and transfer on nitrocellulose paper $(0.2 \mu \mathrm{m}$; BioRad, 160-0112), blotting was performed in 5\% milk with the following antibodies: MMP2 (1:2,000; Abcam, ab92536), MMP14 (1:2,000; Abcam, ab51074), $\gamma$-tubulin (1:10,000; Sigma, T5168). Horseradish peroxidase (HRP)-conjugated antibodies: goat anti-rabbit (HRP) (1:10,000; Stratech, 43C-CB1106-FIT), goat anti-mouse (HRP) (1:10,000; Stratech, 43-GM30-FIT). HRP detection was performed using Clarity Western ECLSubstrate (BioRad,170-5060) and imaged on a gel imager with serial exposure. For the complete gels as imaged see Supplementary Fig. 13. For band intensity quantification see 'Image analysis'.

\section{RNAScope}

The sample was fixed overnight at $4{ }^{\circ} \mathrm{C}$ in DEPC $4 \%$ PFA and then serially dehydrated in increasing concentrations of methanol-PBST(DEPC PBS $+0.1 \%$ Tween $)(25 \%, 50 \%, 75 \%$ and $100 \% ; 10$ min incubation in each concentration). Sample was either stored in $100 \%$ Methanol at $-20^{\circ} \mathrm{C}$ or directly rehydrated in preparation for in situ hybridization. For rehydration the sample was incubated for $30 \mathrm{~min}$ in $100 \%$ methanol $+0.2 \mathrm{M} \mathrm{HCl}$, followed by serial dehydration in methanol + PBST (75\%,50\% and $25 \%)$. After washing the rehydrated sample in PBST + BSA (1\%; Sigma-Aldrich, A3311-50G), it was incubated in Protease Plus (Bio-Techne, 322330) for $30 \mathrm{~min}$ at $40^{\circ} \mathrm{C}$ and rinsed in channel $1(\mathrm{Cl})$ probe. The sample was placed in the probe mixture, which was prepared by diluting channel 2 (C2) and channel 3 (C3) 1:50 in the $\mathrm{C} 1$ probe. The probe mixture was warmed to $40^{\circ} \mathrm{C}$ and allowed to cool to room temperature before the sample was placed in it. After a $2 \mathrm{~h}$ incubation of the sample in the probe mixture at $40^{\circ} \mathrm{C}$, it was washed for $10 \mathrm{~min}$ in $0.5 \times \mathrm{ACD}$ wash buffer (WB; prepared by warming $50 \times \mathrm{WB}$ stock at $40^{\circ} \mathrm{C}$ for $20 \mathrm{~min}$ and diluted 1:100 in DEPC water; Bio-Techne, PN310091) and stored in 5× SSC (prepared from $20 \times$ stock in DEPC water; Thermo Fisher Scientific, 15557044) overnight at room temperature. Sample was then removed from $5 \times$ SSC and washed twice in WB for 10 min each before placing it to AMP1 (Bio-Techne, 320851) for $30 \mathrm{~min}$ at $40^{\circ} \mathrm{C}$. After two 10-min washes in WB the sample was placed in AMP2 for 30 min at $40^{\circ} \mathrm{C}$. For the last step of the amplification, the sample was washed twice for 10 min each in WB and placed in AMP3 for 15 min. To start developing the signal, the sample was placed in HRP-C1 incubated for $15 \mathrm{~min}$ at $40^{\circ} \mathrm{C}$ followed by moving the sample into TSA with diluted fluorophore (used 1:500) and followed by $15 \mathrm{~min}$ incubation at $40^{\circ} \mathrm{C}$ in HRP blocker to stop the reaction. The same procedure was followed for all three channels (HRP-C1, HRP-C2 and HRP-C3).

TSA fluorophores used with respective channel: Opal-570 nm, C1; Opal-650 nm, C2; Opal-520 nm, C3. RNAScope probes used: RNAscope Probe - Mm-Mmp14 (Bio-Techne, 479061), RNAscope Probe - Mm-Mmp2-C2 (Bio-Techne, 315931-C2), RNAscope Probe Mm-Nodal-C3 (Bio-Techne, 436321-C3), RNAscope Probe - Mm-Tdgf1 (Bio-Techne, 506411).

All washes and dehydration-rehydration steps were done in 12-well plates ( $2 \mathrm{ml}$ per well). Protease treatment, amplification and developing signal were done in 96-well plates (4 drops per well).

\section{RT-PCR}

E6.5 embryos were recovered from pregnant mice and the extraembryonic ectoderm was removed and discarded. Then the remaining embryonic part (epiblast + visceral endoderm) was cut in half along the proximal-distal axis, making sure that the long axis of the epiblast was parallel to the plate. Each half was transferred into $20 \mu$ l of extraction buffer from the PicoPure RNA Isolation kit and snap frozen on dry ice. After having been transferred to $-80^{\circ} \mathrm{C}$ overnight, embryos were thawed on ice and incubated at $42{ }^{\circ} \mathrm{C}$ for $30 \mathrm{~min}$. To the $20 \mu \mathrm{l}$ of the lysate, $20 \mu \mathrm{l}$ of $70 \%$ ethanol was added and transferred to a pre-conditioned PicoPure column. Then the column was centrifuged at $100 \mathrm{~g}$ for $2 \mathrm{~min}$ and $14,500 \mathrm{~g}$ for $30 \mathrm{~s}$. Then, $100 \mu \mathrm{l}$ of wash buffer 1 was added and spun at $100 \mathrm{~g}$ for $1 \mathrm{~min}$. DNase was then applied to the column for $30 \mathrm{~min}$ and spun down at $100 \mathrm{~g}$ for $1 \mathrm{~min}$. The column was washed again with wash buffer 1 and spun down at $100 \mathrm{~g}$ before being washed twice with wash buffer 2 (one spin at $100 \mathrm{~g}$ for $1 \mathrm{~min}$ and the second at $14,500 \mathrm{~g}$ for $2 \mathrm{~min}$ ). The columns were centrifuged once more at $14,500 \mathrm{~g}$ for $2 \mathrm{~min}$ to remove residual liquid before eluting the RNA. RNA was eluted by adding $20 \mu \mathrm{l}$ of nuclease-free water and centrifuging into an RNase-free tube for $1 \mathrm{~min}$ at $14,500 \mathrm{~g}$.

For quantitative PCR (qPCR) plate preparation the master mix was prepared from the Power SYBR Green RNA-to $C_{\mathrm{T}}$ 1-Step kit. Quantities were calculated for $10 \mu \mathrm{l}$ per well: $2 \times$ SYBR mix, $125 \times$ enzyme mix, $2 \mu \mathrm{l}$ of RNA sample and nuclease free water. In every well, $5.3 \mu \mathrm{l}$ of primer mix was added made up of $0.3 \mu \mathrm{l}$ of $10 \mu \mathrm{M}$ forward and reverse primers, and $5 \mu$ of nuclease free water.

PCR primers used were: Cerberus (also known as Cer1) forward, 5'-CTCTGGGGAAGGCAGACCT-3'; Cerberus reverse, 5'-CCAC AAACAGATCCGGCTT-3'; MMP25 forward 5'-CCCTGACCTCCT CCAGACTT-3'; MMP25 reverse 5'-GACCTTCGCATCGGGATTCT-3'.

Expression was normalized to the housekeeping gene Gapdh.Primers used: Gapd $h$ forward, 5'-AGGTCGGTGTGAACGGATTTG-3'; Gapdh reverse, 5'-TGTAGACCATGTAGTTGAGGTCA-3'.

To calculate relative expression, the difference of quantification cycles (Cq) between Gapdh and the gene of interest was first calculated for every replicate of the reaction $(\Delta \mathrm{Cq})$. Then the average $\Delta \mathrm{Cq}$ expression (which is $2^{\Delta \mathrm{Cq}}$ ) is calculated and divided by the $\Delta \mathrm{Cq}$ of the Cerberus-high half $(\Delta \Delta \mathrm{Cq})$. Relative gene expression is then calculated based on the differences of $\Delta \Delta \mathrm{Cq}$ between samples.

All primers were validated by performing qPCRs using different concentrations of sample and assessing linearity of Cq difference. In addition, analysis of melting curves showed single amplicon production.

\section{Fluorescent gelatin-degradation assay}

Cy3-gelatin was plated on ibiTreat microscopy plates (Ibidi, 80826) and ES cells were seeded on top at $\sim 5,000$ cells/well. Cy3-gelatin reagents and protocol for coating were provided in the QCM Gelatin Invadopodia Assay Kit (Millipore, ECM671).

\section{Mmp14-knockout ES cell-line generation}

The deletion of Mmp14 was targeted to exon 1 at the translation initiation site. Two gRNAs to flank the initiation codon were designed using the online CRISPR Design Tool (http://tools.genome-engineering.org) 
by inputting the sequence for exon 1 of $M m p 14$. From the list of possible gRNAs designed by the tool the following sequences were selected based on their position on the exon (to flank the initiation codon) and their score of inverse likelihood of off-target binding. The gRNAs selected to flank the initiation codon are: gRNA1: 5'-GTGGATTCCTAGAGCGCGGT-3' and gRNA2: 5'-GGAGGCTGCGGGAGGGTCGA-3'.

Each gRNA was cloned into the plasmid pSpCas9(BB)-2A-Puro (PX459) (deposited in Addgene by the F. Zhang laboratory; Addgene, 62988). Both gRNAs were co-transfected in LifeAct-GFP ES cells using the Lipofectamine 3000 transfection reagent (Thermo Fisher Scientific, L3000001) according to the manufacturer's directions (250 ng of each plasmid).

After two days of antibiotic selection using $2 \mathrm{\mu g} \mathrm{ml}^{-1}$ Puromycin (Invivogen, ant-pr-1), the transfected cells were washed in fresh medium and allowed two more days to recover from the antibiotic treatment. To isolate individual clones, the remaining colonies surviving the antibiotic selection were trypsinized ( $3 \mathrm{~min}$ in Trypsin-EDTA (Life Technologies, 25300054) and stopped with FC medium) and plated at 1 cell per well in two 96-well plates. The plates were checked every day until colonies became visible. Out of 192 wells, 48 had a single colony growing. Each of the 48 wells was trypsinized and split into 3 new wells in 3 different 96-well plates to create triplicates of all clones. Once the colonies in the new plates became confluent, two plates were frozen down by first trypsinizing and inactivating, followed by addition of equal volume of freezing medium (FBS (Stem Cell Institute) + 20\% DMSO (Sigma-Aldrich, D2650). The cells in the remaining plate were allowed to grow for two more days before extracting DNA from each clone and genotyping.

Lysis of cells to extract DNA was performed using the following protocol: Medium was removed from the wells and replaced with $250 \mu \mathrm{l}$ of TNES (50 $\mu \mathrm{M}$ Tris (pH7.4), $100 \mu \mathrm{M}$ EDTA (pH8.0), $400 \mathrm{mM} \mathrm{NaCl}$, $0.5 \% \mathrm{SDS})+5 \mu \mathrm{l}$ of proteinase K (Qiagen, 19131). Cells were detached by pipetting up and down and transferred to a $1.5-\mathrm{ml}$ tube followed by incubation at $55^{\circ} \mathrm{C}$ for $30 \mathrm{~min}$. To precipitate cell membrane $75 \mu \mathrm{l}$ of $6 \mathrm{M} \mathrm{NaCl}$ was added and vigorously shaken for $30 \mathrm{~s}$ before spinning for $10 \mathrm{~min}$ at $16,900 \mathrm{~g}$. A $1: 1$ volume of $100 \%$ ethanol was added to the supernatant and inverted 20 times before spinning down for $5 \mathrm{~min}$ at maximum speed at $4{ }^{\circ} \mathrm{C}$. The supernatant was then discarded, and the pellet was washed with $70 \%$ ethanol and span down again for $5 \mathrm{~min}$ at maximum speed at $4{ }^{\circ} \mathrm{C}$. The supernatant was removed again and left the tubes to air dry for $10 \mathrm{~min}$ at room temperature. To dissolve the DNA pellet, $20 \mu$ l of DNase-free water was added and incubated at $65^{\circ} \mathrm{C}$ for 20 min. From the resulting DNA sample, $2 \mu \mathrm{l}$ were used per PCR reaction.

For genotyping the primers used were: forward primer, 5'-CAGTTCGCCGACTAAGCAGA- 3'; reverse primer, 5'-TACTTCGGG GCTGAAGTTGC-3'.

\section{Imaging}

A Leica SP8 inverted confocal microscope was used to image fixed embryos. For live imaging the same set up was used with laser power not exceeding $2.5 \%$ of that of the solid-state Ti:Sapphire laser. Lifeact-GFP, T-GFP and CellEvent Caspase-3/7-treated embryos were imaged using 488-nm excitation. For maximum intensity projections of basement membrane, laminin, collagen IV or perlecan were imaged with optical slicing at $0.6 \mu \mathrm{m}$ and projected using the Fiji function ' $Z$ project'.

\section{Image analysis}

Images were acquired using the Leica software LASX and were processed and analysed on Fiji image processing software (ImageJ 1.52h) or Imaris 9.1.2 for 3D reconstruction and 3D analysis. For perforations size quantification, binary images were generated from MIP images of basement membrane. Subsequently, the perforations were identified and their size was extracted using the Fiji function 'particles'. To establish correlation between perforations domain and AVE position, a maximum projection image was used to determine the area of AVE coverage based on signal from Cerberus. Then a binary image of maximum-intensity-projected basement membrane was created and the particles function in Fiji was used to calculate total area covered by perforations in the anterior or posterior domain (as indicated by area of AVE coverage). Similarly, for gelatin-degradation assay a binary image of maximum-intensity-projected Cy3-gelatin was generated and percentage of area absent in the total area of the field of view was calculated. For western blot band analysis the 'gel analysis' Fiji tool was used. The bands being quantified were manually selected and an intensity plot was generated. The area under the plot, representing total intensity, was measured using the magic wand tool. Protein bands of interest were normalized against the total intensity of the respective tubulin band and compared. For vector mapping, the Fiji plugin 'orientationj' was used. Maximum-intensity-projected images of basement membrane were used to generate vector maps using this plugin. The vector angles were exported and used to create histograms to assess skewness. For 3D analysis of gastrulated embryos in the presence or absence of MMP inhibitors, Imaris was used to detect cells with the spot detection function and allocate them manually based on their relative position to the basement membrane. For presentation purposes, the basement membrane was surface rendered in Fig. 4l, to visually demonstrate position of anterior primitive streak, using the surface render Imaris function. To quantify mRNA levels in control SB431542-treated and $\mathrm{Noda}^{t / f l t} \mathrm{CreER}^{T 2}+$ tamoxifen embryos, in toto images were acquired with $Z$-compensation function. The individual signal spots per embryo were automatically detected by the spot detection function of Imaris software using a prediction for the spot diameter at $2 \mu \mathrm{m}$. Spot detection quality was adjusted and curated manually.

\section{Single-cell sequencing analysis}

Re-analysis of the GSE109071 dataset ${ }^{21}$ was performed using R (v.3.4.4) and Seurat (v.3.0.1). The matrix of read counts was input into Seurat, normalized (log), and scaled. Three thousand variable genes were used to identify clusters. Dimensional reduction was performed using UMAP. Cell identifiers were parsed from the GEO entry and added to the Seurat Object using (make_sample2Age_table.sh). All expression values are $\log$ (counts). Marker genes were used to identify the epiblast (Pou5f1), extraembryonic ectoderm (Bmp4) and visceral endoderm (Amn) cells in the UMAP. The matrix of RPKM values from GSE109071 was used to calculate gene expression correlation between pairs of selected genes. epiblast cells at ages 6.25 and 6.5 were extracted from the matrix and Pearson's correlation coefficient $(R)$ with $P$ value are given for each comparison. The points are coloured by density using a kde2d kernel. Scripts and data to recreate analysis and figures are available from https://github.com/darogan/Kyprianou_Zernika-Goetz.

\section{ChIP-seq analysis}

ChIP tracks for Smad2/3 from GSE70486 ${ }^{25}$ in TDF (tiled data file) format were visualized in Integrative Genomics Viewer ${ }^{33}$ and signal intensities were normalized against $1 \times 10^{6} /$ total reads to give reads per million (RPM).Scripts to recreate ChIP-seq analysis and figures can be found at https://github.com/darogan/Kyprianou_Zernika-Goetz. Analysis was performed using $\mathrm{R}$ (v.3.4.4).

\section{Statistics}

Statistical analyses were performed in GraphPad Prism 6.0. Quantitative data are presented as the mean \pm s.e.m. Quantitative data were analysed using a two-sided unpaired Student's $t$-test and qualitative data were analysed with $\chi^{2}$ tests. To compare the distributions of vector angles, the Kolmogorov-Smirnov test was used. Unless otherwise noted, each experiment was performed at least three times. Circular statistics in Fig. $4 \mathrm{~F}$ were generated using the circular statistics software Oriana.

\section{Reporting summary}

Further information on research design is available in the Nature Research Reporting Summary linked to this paper. 


\section{Article}

\section{Data availability}

The source data used in all graphs are provided in the Source Data files. Raw image files are available from the corresponding author upon request.

\section{Code availability}

The codes used in this study are available at https://github.com/ darogan/Kyprianou_Zernika-Goetz and https://doi.org/10.5281/ zenodo.3610335.

33. Robinson, J. et al. Integrative genomics viewer. Nat. Biotechnol. 29, 24-26 (2011).

Acknowledgements We thank D. Glover, M. Shahbazi and M. Zhu for valuable discussions; A. Cox for drawing the model in Fig. 4i; M. Kuehn for the Nodal ${ }^{f / f l}$ mice and V. Kouskoff for the
T-GFP mice. D.S.B. is supported by the FNRS; W.N. is supported by WELBIO; I.M. is a FNRS research associate and an investigator of WELBIO. The M.Z.-G. laboratory is supported by grants from the European Research Council (669198) and the Wellcome Trust (098287/Z/12/Z)

Author contributions C.K. and N.C. designed and carried out the experiments and data analysis. R.S.H. performed the bioinformatics analysis. G.A. performed the RNA in situ experiments. W.N., D.S.B. and I.M. generated the Ttr-cre;Rhoa ${ }^{f /-}$ embryos. M.Z.-G., C.K. and N.C. conceived the study and wrote the manuscript. M.Z.-G. supervised the study.

Competing interests The authors declare no competing interests.

Additional information

Supplementary information is available for this paper at https://doi.org/10.1038/s41586-020 2264-2.

Correspondence and requests for materials should be addressed to M.Z.-G.

Peer review information Nature thanks Elizabeth Lacy, Patrick Tam and Kenneth Yamada for their contribution to the peer review of this work.

Reprints and permissions information is available at http://www.nature.com/reprints. 

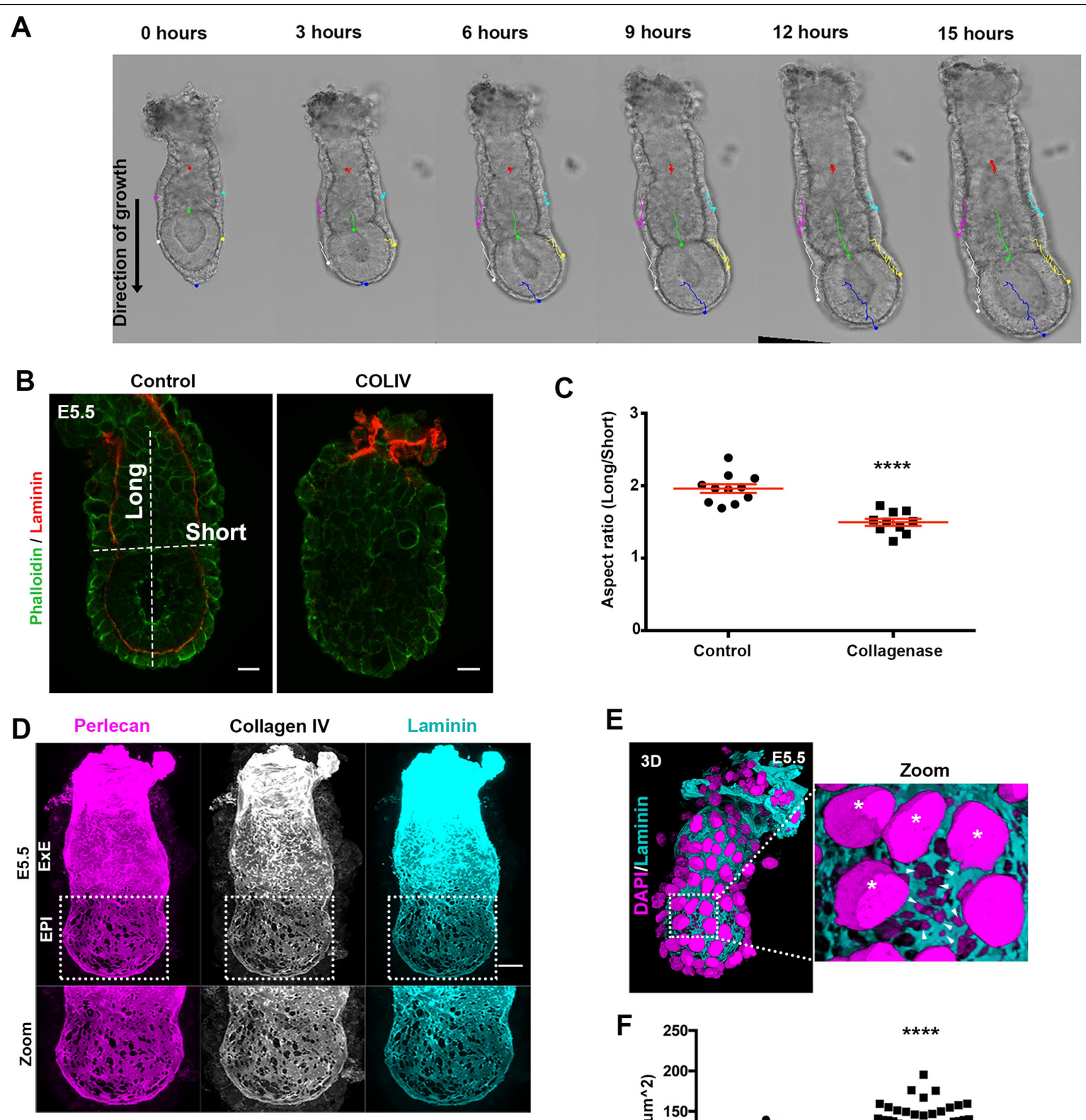

E
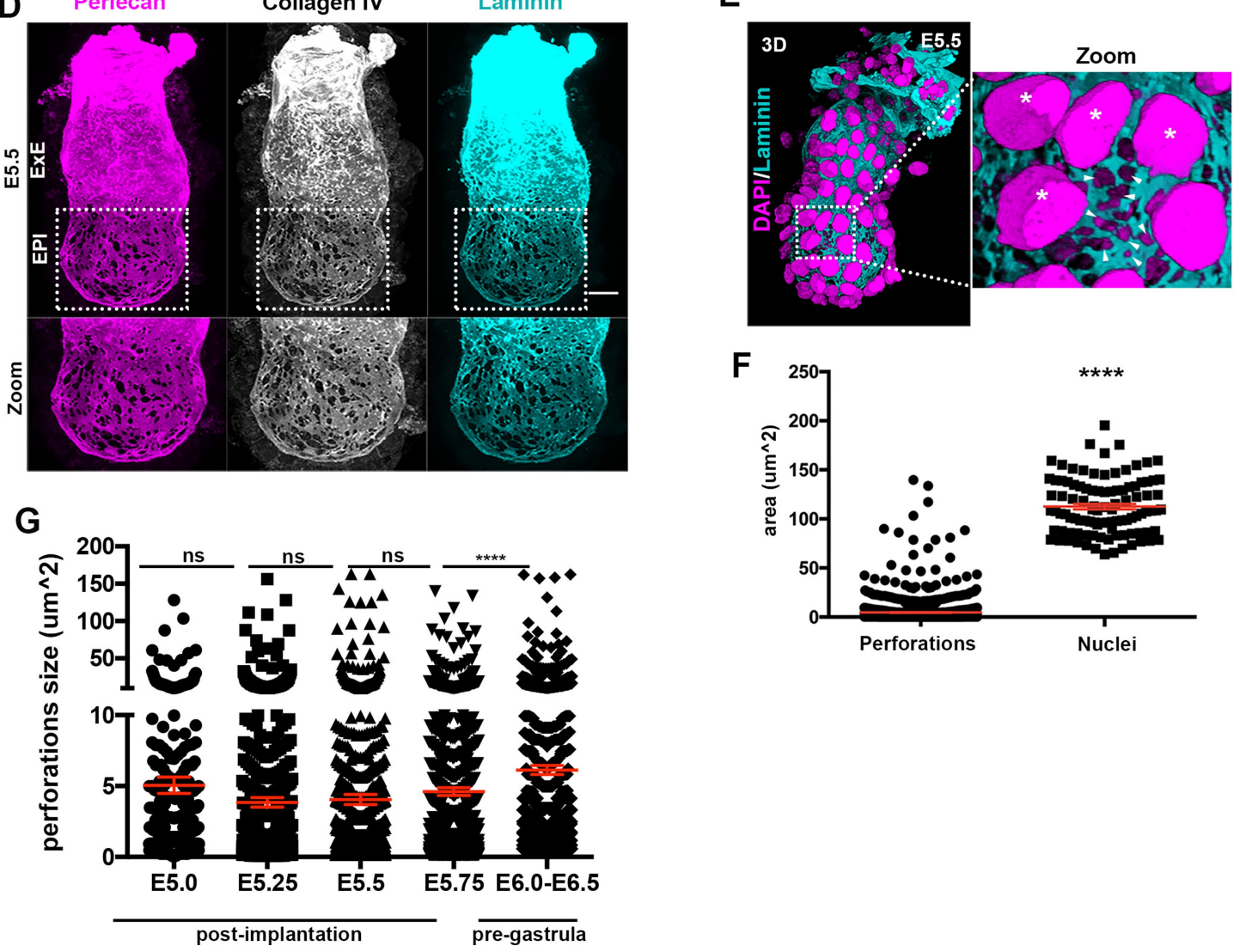

Extended Data Fig. 1 See next page for caption. 


\section{Article}

Extended Data Fig. 1 | Post-implantation embryo growth and basement membrane morphology. a, Tracked bright-field stills from a time-lapse video of an E5.5-E5.75 embryo showing egg-cylinder growth along the embryo proximal-distal axis. Tracks are arbitrarily colour-coded and follow growth in $x y$ dimensions of specific anatomical characteristics. Red, ectoplacental coneextraembryonic ectoderm boundary; magenta-cyan, extraembryonic ectoderm; green, epiblast-extraembryonic ectoderm boundary; whiteyellow, epiblast; blue, distal epiblast. $n=5$ embryos. b, E5.5 embryos cultured for $8 \mathrm{~h}$ in the presence or absence of collagenase IV.c. Egg-cylinder aspect ratio comparison in control and collagenase IV-treated embryos. Unpaired Student's $t$-test; ${ }^{* * * *} P<0.0001$; mean \pm s.e.m. In $\mathbf{b}$ and $\mathbf{c}, n=11$ (control) and 11 (collagenase IV-treated) embryos. d, Representative example of an E5.5 embryo stained for different components of basement membrane. Basement membrane perforations can be identified with all markers. $n=10$ embryos.

e, Representative examples of E5.5 embryos $(n=20)$ showing the size of basement membrane perforations relative to the size of nuclei. Asterisks, visceral endoderm nuclei; arrowheads, basement membrane perforations. f, Quantification of basement membrane perforations and cell nuclei area. $n=1,501$ perforations and 100 nuclei from 6 embryos. Two-sided unpaired Student's $t$-test; ${ }^{* * * *} P<0.0001$; mean \pm s.e.m.g, Quantification of basement membrane perforation size during post-implantation development. Average perforation size remains the same in early post-implantation stages but increases in the pre-gastrula stages. $n=423$ (E5.0), $n=1,046(\mathrm{E} 5.25), n=1,327$ (E5.5), $n=1,501$ (E5.75), $n=1,615$ (E6.0-E6.5). One way ANOVA; ${ }^{* * *} P<0.0001$ mean \pm s.e.m. Scale bars, $20 \mu \mathrm{m}$ 

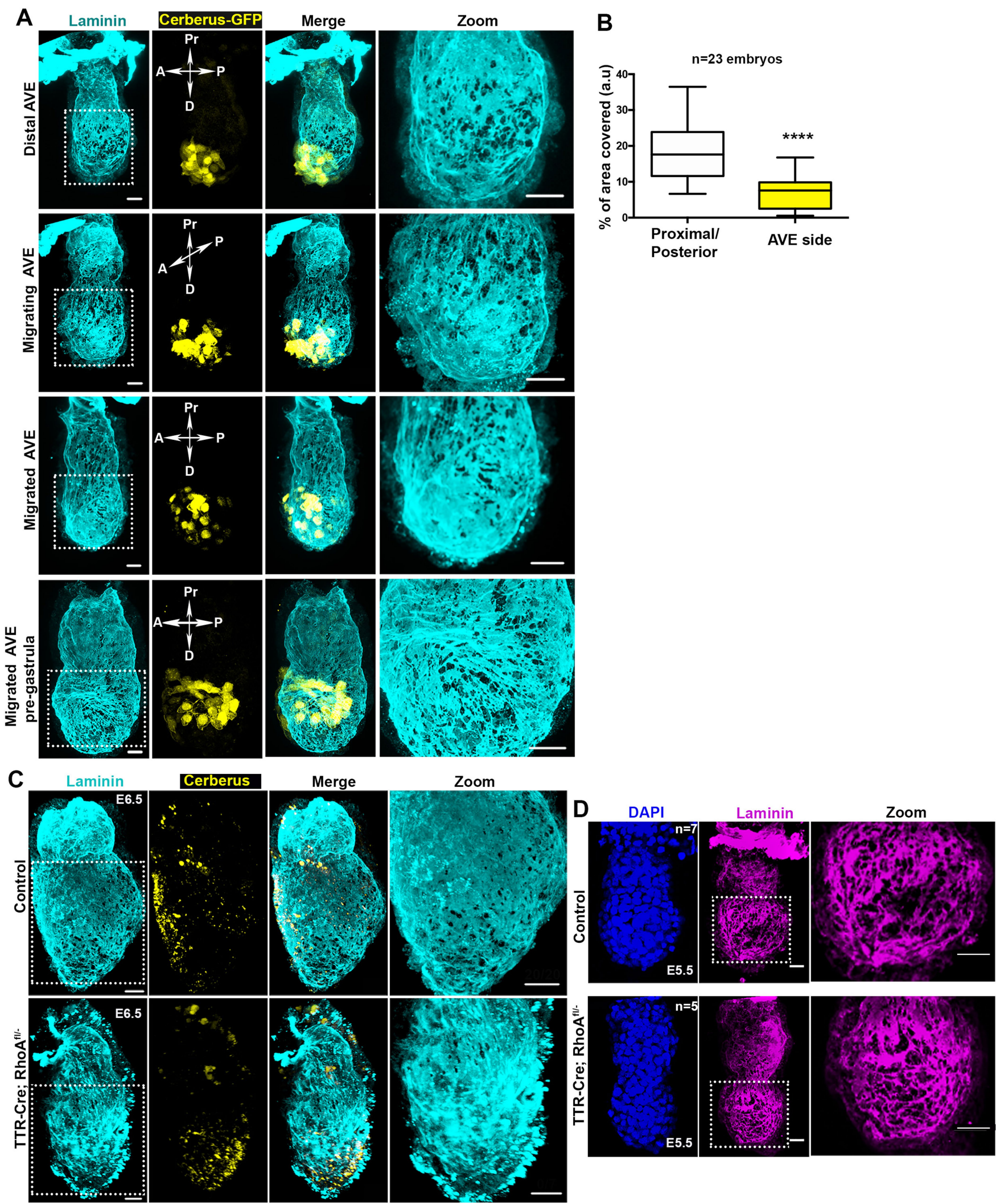

\section{Extended Data Fig. 2 |AVE migration regulates the distribution of} basement membrane perforations. a, AVE position and basement membrane remodelling using transgenic Cerberus-GFP embryos for AVE identification. $n=23$ embryos. b, Quantification of basement membrane area covered by perforations at regions away (proximal or posterior) or close to the AVE side as in Fig. 2b, with embryos from different AVE migration stages pooled together. Two-sided unpaired Student's $t$-test; ${ }^{* * *} P<0.0001$. In box plots, centre lines show median values, box limits represent the upper and lower quartiles, and whiskers show the range of values. c, Representative examples of E6.5 pre-gastrula control ( $n=20$ embryos) and Ttr-cre; Rho $a^{f l-}$ embryos $(n=7$ embryos). Blocking AVE migration results in abnormal distribution of basement membrane perforations. d, Representative examples of control $(n=5)$ and Ttr-cre; Rho $a^{f l /-}(n=5)$ E5.5 embryos. Scale bars, $20 \mu \mathrm{m}$. 


\section{Article}
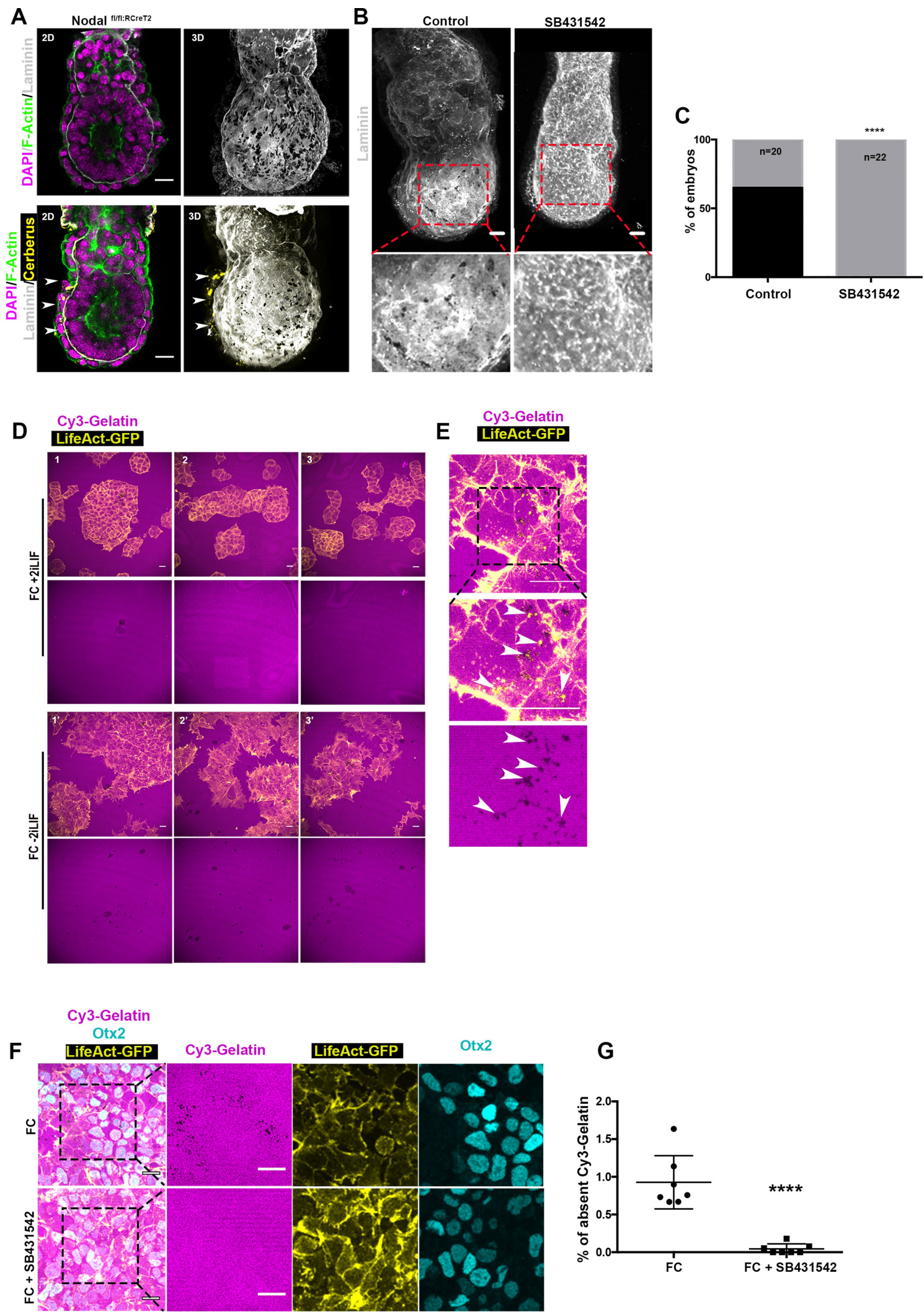

Extended Data Fig. 3 | See next page for caption. 
Extended Data Fig. 3 Nodal controls basement membrane remodelling in embryos and ES cells. a, Representative examples of control Nodal $t^{f l f l} \mathrm{CreER}^{T 2}$ embryos ( $n=8$ embryos) fixed and stained immediately upon recovery. In the absence of tamoxifen treatment, Nodal ${ }^{f l f l} \mathrm{CreER}^{T 2}$ embryos have a normal basement membrane appearance, as Nodal expression is not affected. Bottom, position of AP axis and basement membrane perforation patterning.

Arrowheads, AVE. b, Representative examples of control embryos $(n=20)$ and embryos treated with Nodal inhibitor $(n=22)$. Note the absence of basement membrane perforations and the appearance of accumulated fibrillar laminin in embryos treated with Nodal inhibitor (SB431542). c, Percentage of embryos with basement membrane perforations appearance in control embryos and embryos treated with Nodal inhibitor (SB431542). $X^{2}$ test; ${ }^{* * * *} P<0.0001$. d, LifeAct-GFP ES cells cultured in conditions to maintain pluripotency
(FC+2i-LIF) and conditions to induce exit from pluripotency (FC-2i-LIF) plated on Cy3-gelatin; three examples for each condition $\left(1,2,3\right.$ and $1^{\prime}, 2^{\prime}$ and $\left.3^{\prime}\right)$. The stripy appearance of gelatin is a result of the way it is plated. Three independent experiments. e, Representative example from three independent experiments of Cy3-gelatin remodelling by LifeAct-GFP ES cells upon exit from pluripotency. Arrowheads point to actin enrichment (invadopodia-like structures) colocalizing with region of remodelled ECM.f, Control and SB431542-treated ES cells plated on Cy3-gelatin and cultured in conditions to allow exit from pluripotency. Right, magnified images showing colocalization of control ES cells with Cy3-gelatin perforations.g, Quantification of results in f, showing amount of $\mathrm{Cy} 3$-gelatin remodelling based on the percentage of fluorescence that is absent. $n=7$ regions for each condition. Two-sided unpaired Student's $t$-test; ${ }^{* * * *} P<0.0001$; mean \pm s.e.m. Scale bars, $20 \mu \mathrm{m}$. 
A

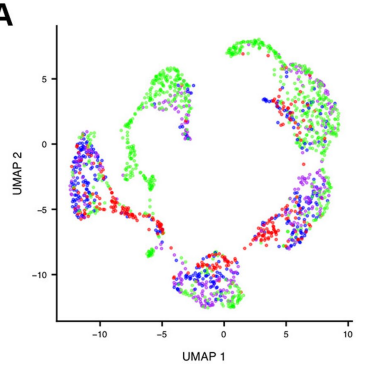

B
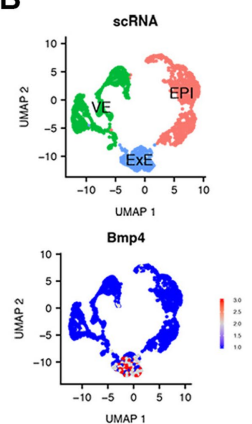

E5.25-E6.5

C

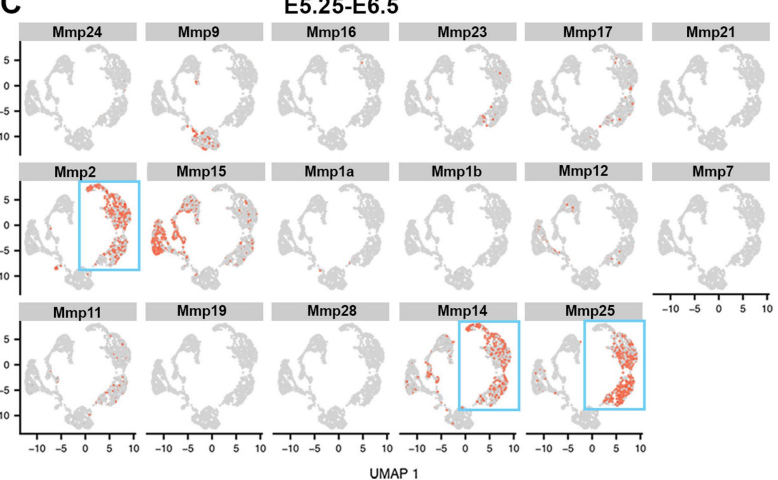

D
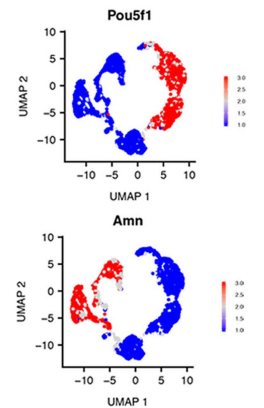

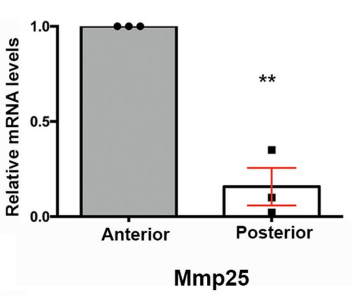

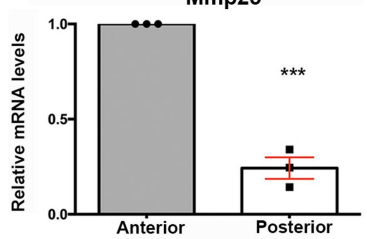

E

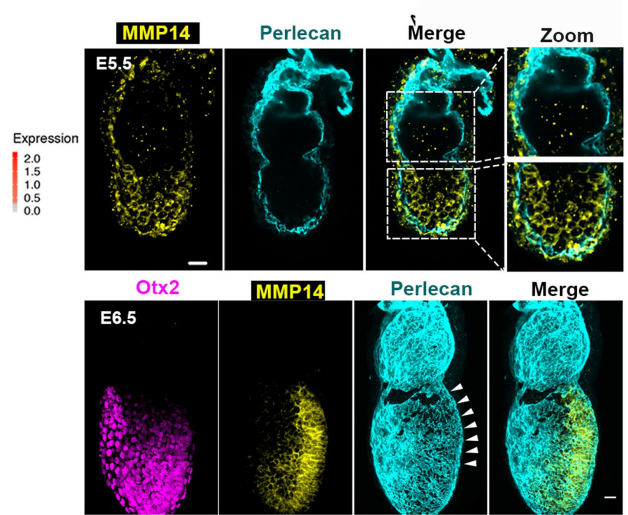

$\mathbf{F}$
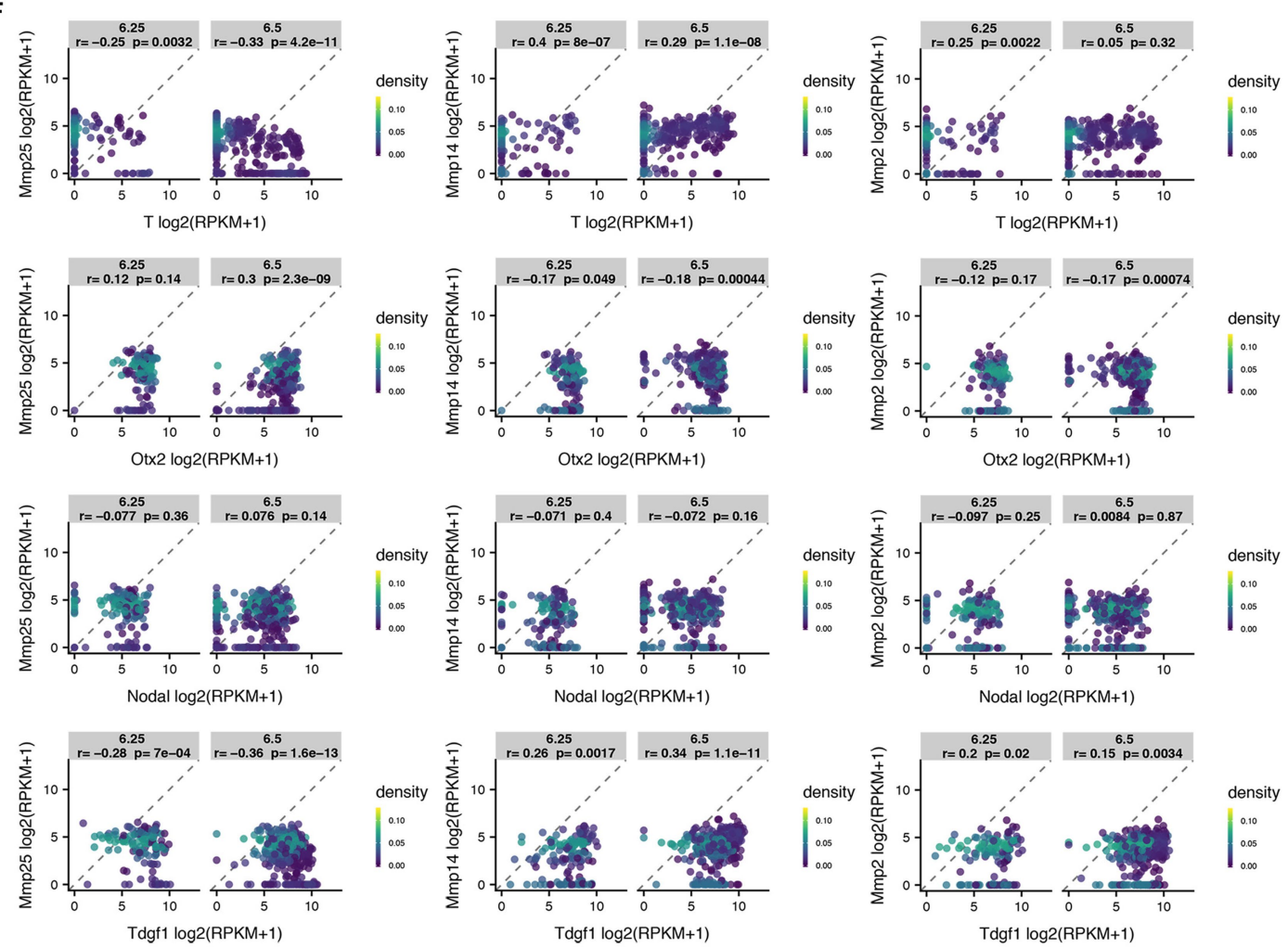

Extended Data Fig. 4 | See next page for caption. 
Extended Data Fig. 4 | MMP expression profiles. a, UMAP showing distribution of cells by embryonic age. $n=1,724$ cells; $n=331$ (E5.25), 269 (E5.5), 321 (E6.25), 803 (E6.5) cells.b, Marker genes identify epiblast (Pou5f1), extraembryonic ectoderm (Bmp4) and visceral endoderm ( $A m n$ ) cells in the UMAP. $n=775$ epiblast cells, 283 extraembryonic ectoderm cells and 666 visceral endoderm cells. c, Expression levels of MMP genes on UMAP plot for E5.25-E6.5. Expression values are $\log$ (counts). Blue box, epiblast. d, mRNA levels of Cerberus and $\mathrm{Mmp} 25$ at the anterior and posterior side of the embryo. Anterior and posterior identity was defined based on Cerberus expression. Mmp25 is highly expressed at the anterior side of the embryo. $n=3$ embryos.
Two-sided unpaired Student's $t$-test. Cerberus: ${ }^{* *} P=0.001 ;$ Mmp25:

${ }^{* * *} P=0.0002$; mean \pm s.e.m. e, E5.5 and E6.5 pre-gastrula embryos stained for MMP14. White arrowheads, prospective primitive streak. $n=10$ embryos for each stage. Scale bars, $20 \mu \mathrm{m}$.f, The matrix of reads per kilobase of transcript per million mapped reads (RPKM) from GSE109071 was used to calculate gene-expression correlation between pairs of genes. Epiblast cells at E6.25 and E6.5 ( $n=527$ cells) were extracted from the matrix and Pearson's correlation coefficient $(R)$ with two-sided $P$ value are given for each comparison. The points are coloured by density (kde2d). 


\section{Article}

A

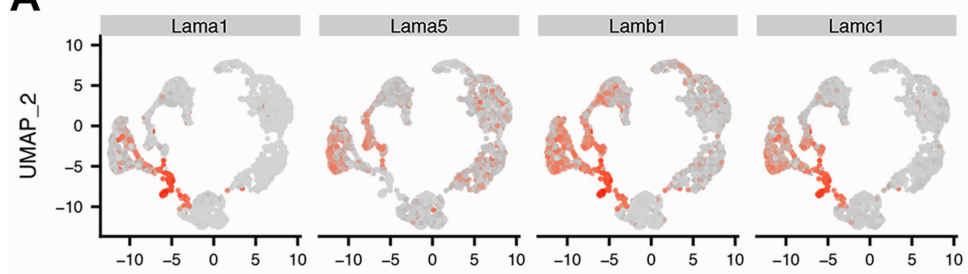

UMAP_1
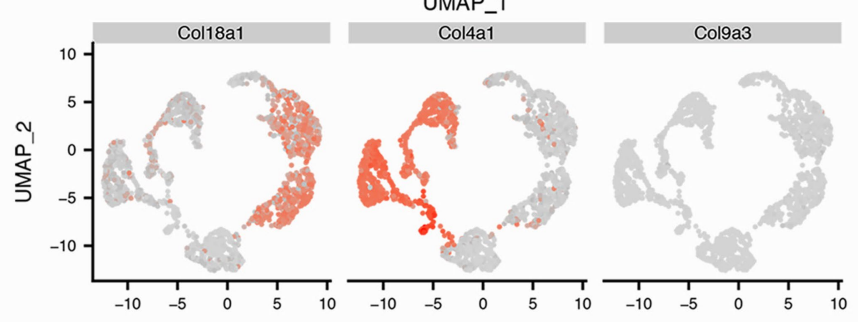

UMAP_1

C
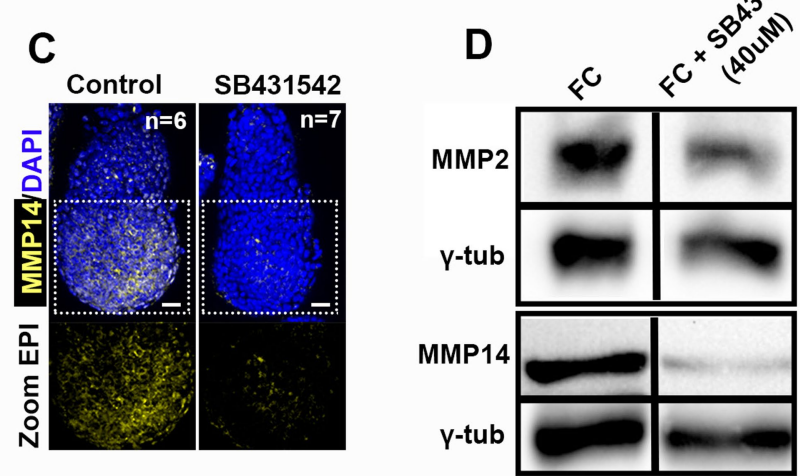

B

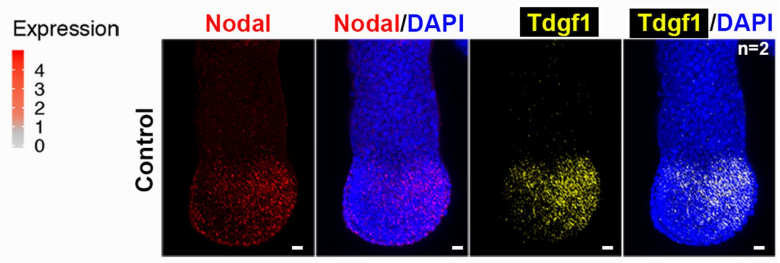

Expression

5

4
3
2
1
0

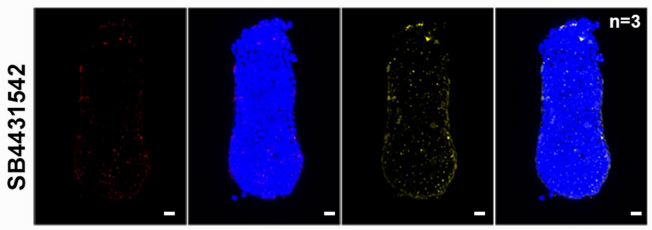

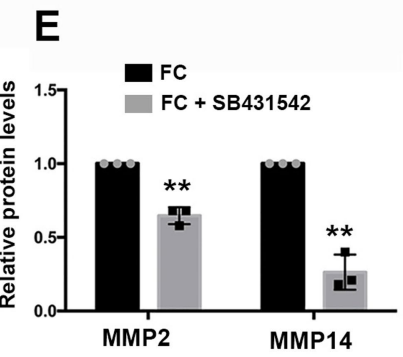

$\mathbf{F}$

chr8

$72 \mathrm{~kb}$
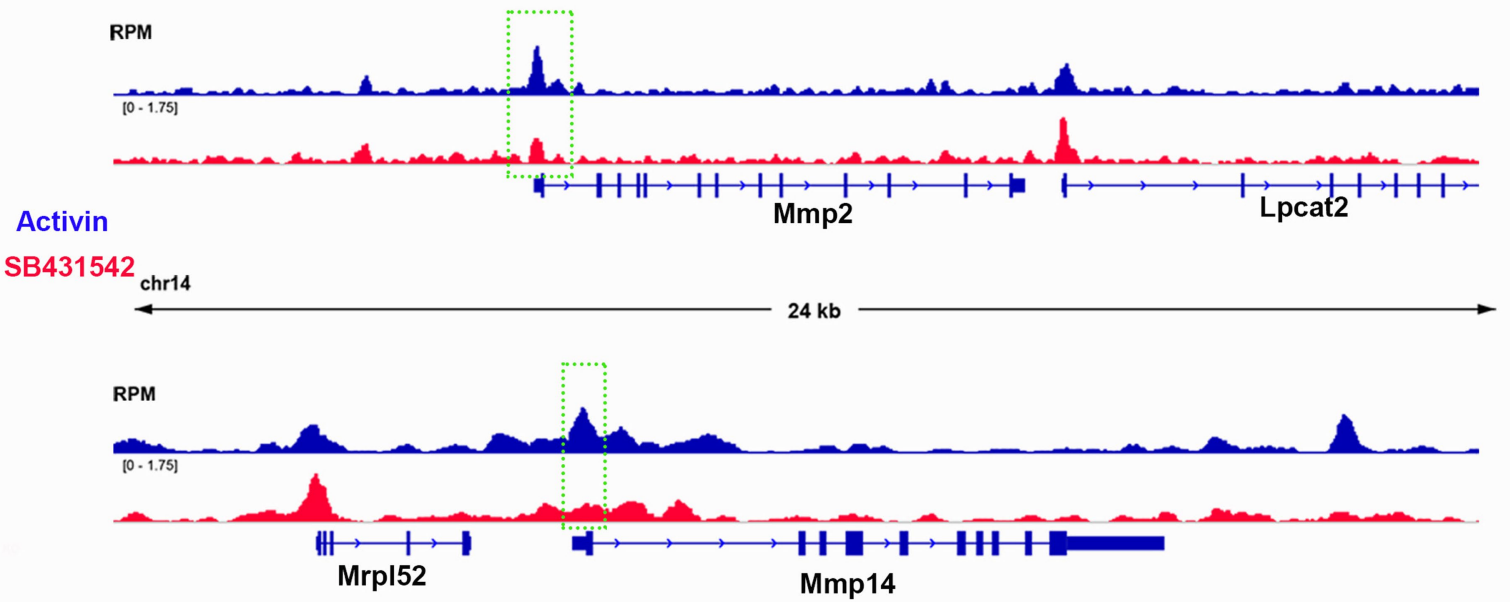

Extended Data Fig. 5 | MMP2 and MMP14 expression is regulated by Nodal. a, Expression levels for ECM genes are plotted on the UMAP for all cell types from E5.25-E6.5 ( $n=1,724$ cells). All expression values are shown as log(counts). b, Representative examples of FISH for control $(n=3)$ and SB431542-treated embryos $(n=3)$. Embryos were recovered at $\mathrm{E} 5.75$ and cultured for $18 \mathrm{~h}$ before fixation. Nodal signalling is inhibited after treatment with SB431542, as shown by the absence of Nodal and Tdgf 1 mRNA.c, Representative examples of control $(n=5)$ and Nodal inhibitor-treated $(n=5)(18 \mathrm{~h})$ embryos stained for
MMP14.d, Western blot analysis for MMP2 and MMP14 in control and Nodal-inhibited (SB431542) ES cells. $n=3$ independent experiments. For gel source data, see Supplementary Fig. 1.e, Quantification of relative protein levels based on western blots of ES cells in the presence or absence of the Nodal inhibitor SB431542. Two-sided unpaired Student's $t$-test. MMP2, ${ }^{* *} P=0.0088$; MMP14, ${ }^{* *} P=0.0086 ; n=3$ independent experiments; mean \pm s.e.m.f, ChIP tracks for Smad2/3. Smad2/3 binding on MMP2 and MMP14 is lost upon Nodal inhibition (green rectangles). Scale bars, $20 \mu \mathrm{m}$. 
A

MMP14
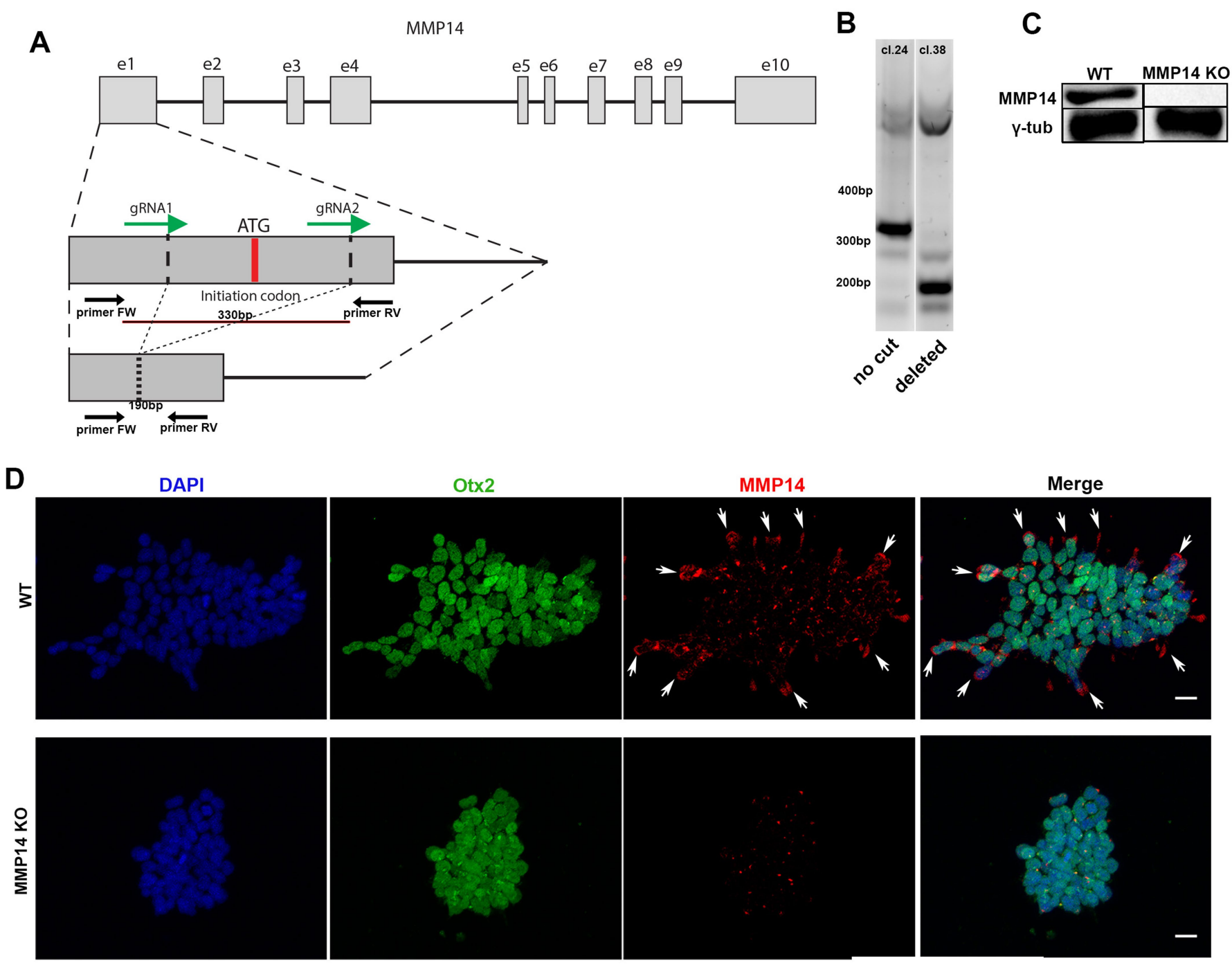

\section{E \\ Cy3-Gelatin}

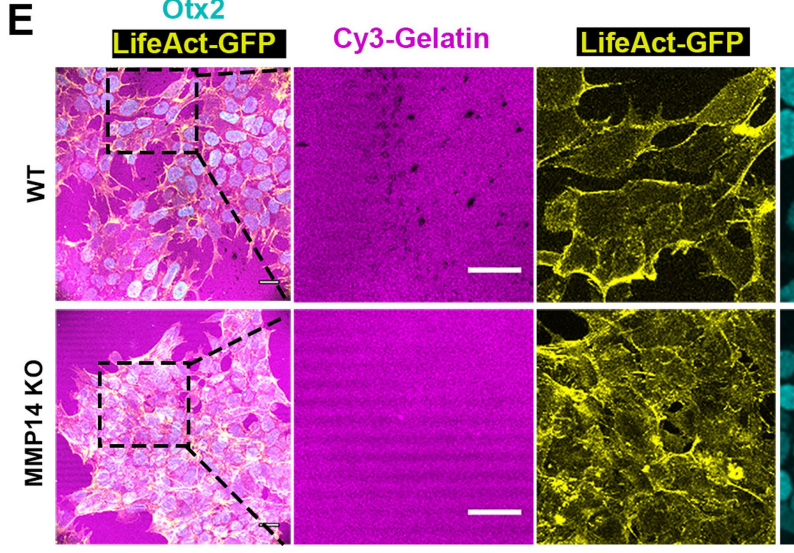

$\mathbf{F}$

Extended Data Fig. 6 | MMP14 is necessary for basement membrane remodelling in ES cells. a, CRISPR-Cas 9 exon 1 ATG initiation codon-deletion strategy. gRNAs designed to flank a 330-bp region that includes the ATG initiation codon. Genotyping primers positions are shown as arrows below the exon region. b, Genotyping results of two clones: in one, the exon remained uncut (clone 24), and in the other (clone 38), it was successfully cut.c, Western blot for MMP14 in clone 38 shows successful MMP14 protein depletion. For gel source data see Supplementary Fig. 1.d, Representative examples of control and Mmp14-knockout (KO) Lifeact-GFPES cells stained for MMP14. MMP14 protein is not detected in the Mmp14-knockout cells. Three independent experiments.e, Representative examples of control and Mmp14-knockout Lifeact-GFP ES cells plated on Cy3-gelatin. Right, magnified images showing colocalization of control and wild-type ES cells with Cy3-gelatin perforations. Note the defective extracellular matrix remodelling in MMP14-knockout ES cells. The striped appearance of gelatin is a result of how it is plated. Three independent experiments. $\mathbf{f}$, Quantification of Cy3-gelatin remodelling in $\mathbf{e}$ based on the percentage of absent fluorescence. Two-sided unpaired Student's $t$-test; ${ }^{* * *} P<0.001 ; n=5$ regions for FC and 7 regions for $M m p 14$ knockout; mean \pm s.e.m. Scale bars, $20 \mu \mathrm{m}$. 


\section{Article}

A Control

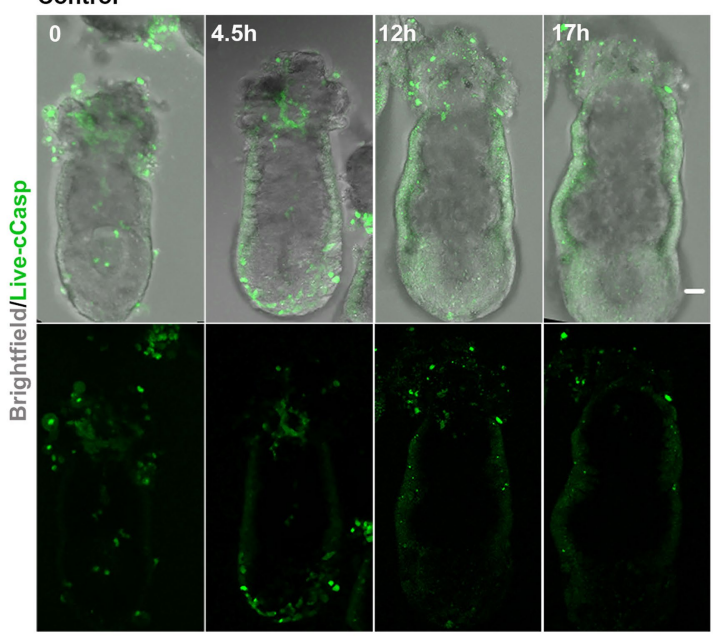

B 0 hours 18 hours

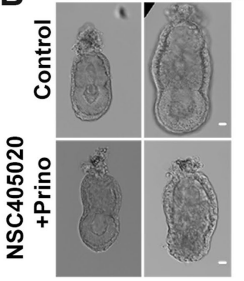

D Control

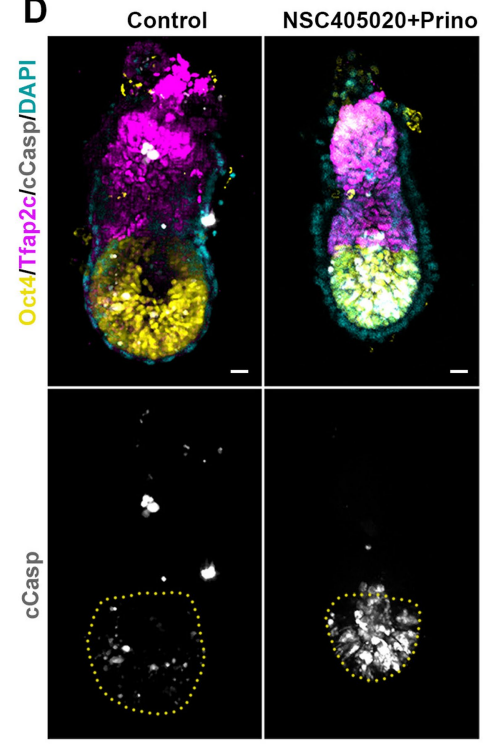

E

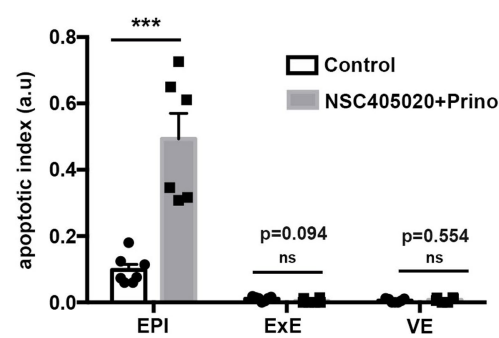

C

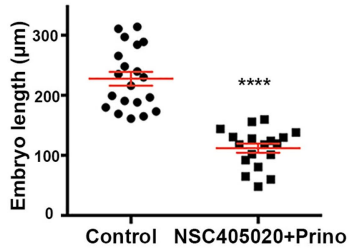

$\mathbf{F}$
NSC405020+Prino

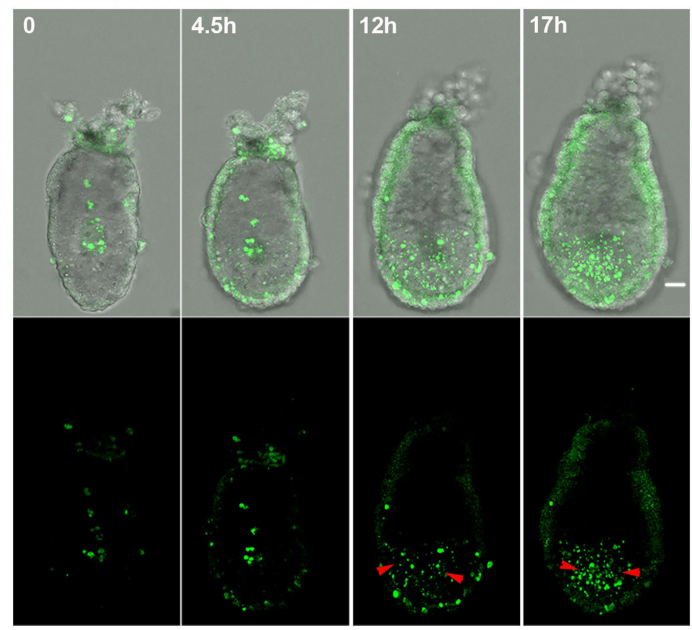

Control

NSC405020+Prino

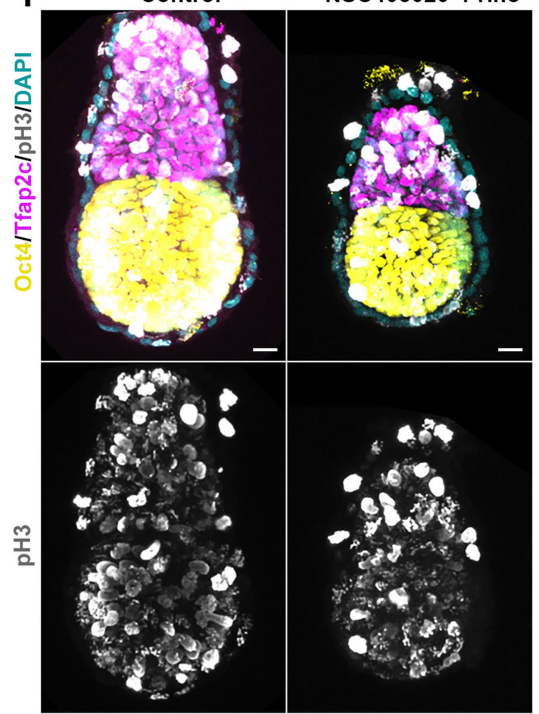

G $\mathrm{p}=0.923 \square$ Control

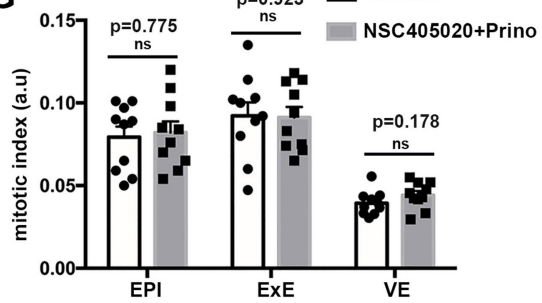

Extended Data Fig. 7 |See next page for caption. 
Extended Data Fig. 7 | MMP activity is indispensable for proper embryo growth. a, Stills from time-lapse video of representative control $(n=5)$ and MMP inhibitor-treated $(n=4)(100 \mu \mathrm{M} \mathrm{NSC} 405020+20 \mu \mathrm{M}$ prinomastat $)$ embryos cultured from $\mathrm{E} 5.75$ for $18 \mathrm{~h}$. Red arrowheads show cell death initiation after growth restriction in the absence of MMP activity. b. Control $(n=20)$ and MMP inhibitor-treated $(n=18)$ embryos ( $18 \mathrm{~h})$. c, Quantification of embryo length (18 h of culture). $n=20$ control and 18 MMP-inhibitor-treated embryos; two-sided unpaired Student's $t$-test; ${ }^{* * * *} P<0.0001$; mean \pm s.e.m.

d, Representative examples of control $(n=7)$ and MMP inhibitor-treated $(n=6)$ embryos stained for the apoptotic marker cleaved caspase-3 (c-Casp3). e, Quantification of apoptotic index (number of apoptotic cells per total cell number). $n=7$ control and 6 MMP inhibitor-treated embryos. Two sided unpaired Student's $t$-test; ${ }^{* * *} P=0.0002$; mean \pm s.e.m.f, Representative examples of control $(n=10)$ and MMP inhibitor-treated $(n=10)$ embryos stained for the mitotic marker phosphorylated histone $\mathrm{H} 3$ (Ser10). g, Quantification of mitotic index (number of mitotic cells per total cell number). $n=10$ control and $10 \mathrm{MMP}$ inhibitor-treated embryos. Two-sided unpaired Student's $t$-test; mean \pm s.e.m. Scale bars, $20 \mu \mathrm{m}$. 


\section{Article}

A

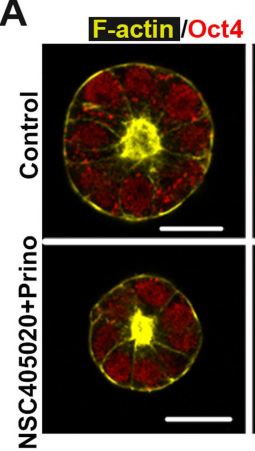

B
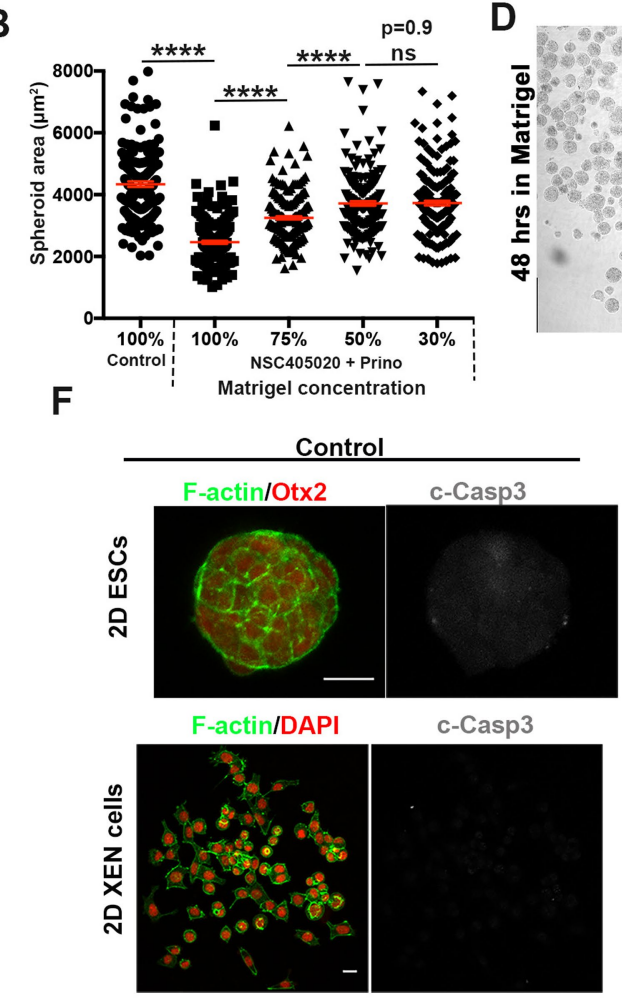

D

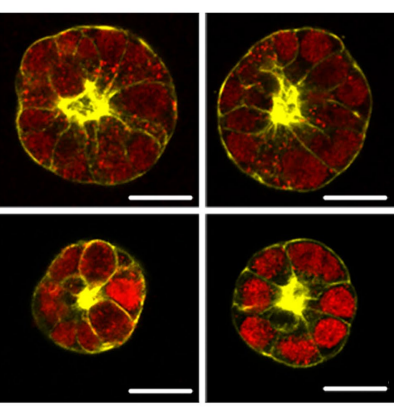

\section{C}

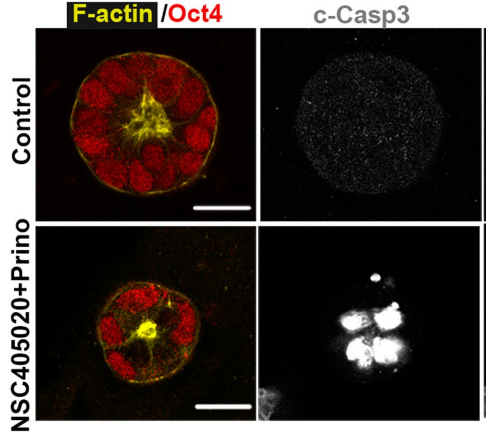

MMP14-KO
WT

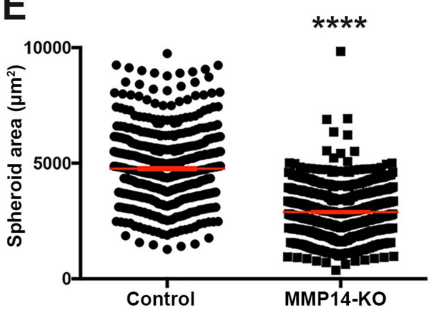

G

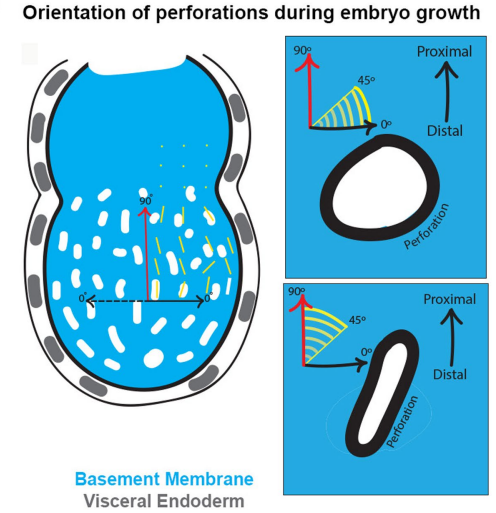

H

Laminin + Vectors

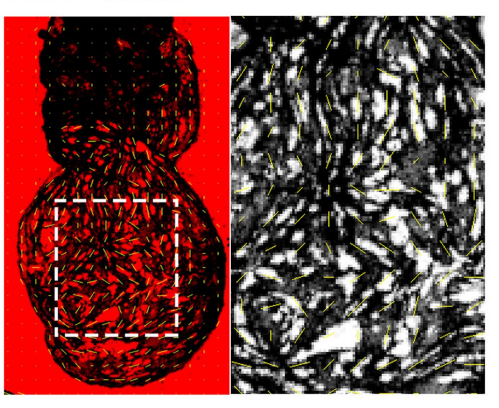

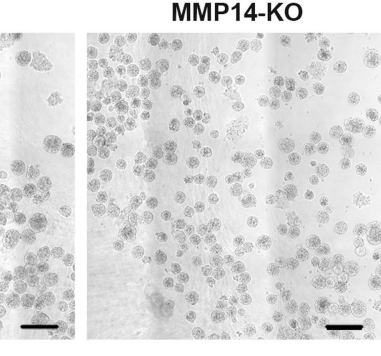

E

Merged

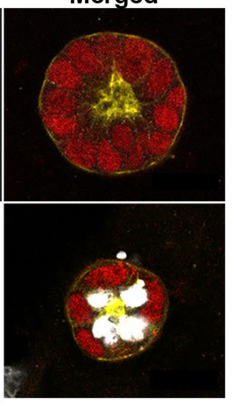

NSC405020+Prino

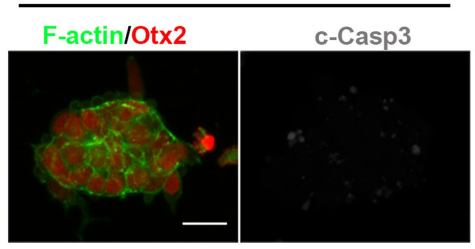

c-Casp3

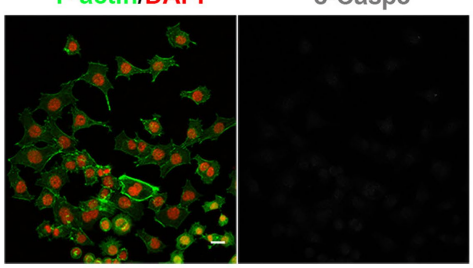

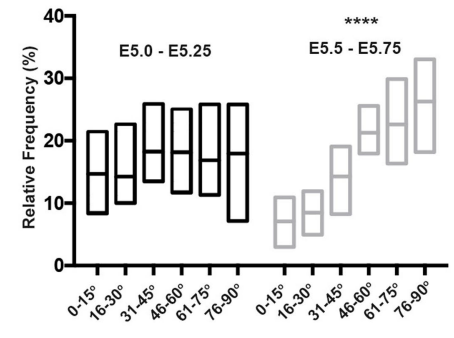

Extended Data Fig. 8 |See next page for caption. 
Extended Data Fig. 8 | Basement membrane remodelling is necessary for ES cell-spheroid growth. a, Representative examples of control and MMP inhibitor $(100 \mu \mathrm{M} \mathrm{NSC} 405020+20 \mu \mathrm{M}$ prinomastat)-treated (treatment started at $48 \mathrm{~h}$ after removal of $2 \mathrm{i}$-LIF for $18 \mathrm{~h}$ ) ES cell spheroids in Matrigel. $n=3$ independent experiments. b, Quantification of ES cell-spheroid area in control and NSC405020 + prinomastat conditions. Reduction of Matrigel concentration results in partial rescue of the phenotype produced upon inhibition of MMP activity. Two-sided unpaired Student's $t$-test; ${ }^{* * * *} P<0.0001$; mean \pm s.e.m. $n=200$ spheroids for each condition.c, Examples of control and NSC405020 + prinomastat-treated (treatment at $48 \mathrm{~h}$ ) ES cell spheroids stained for cleaved caspase-3 (c-Casp3) to monitor apoptosis. $n=3$ independent experiments. $\mathbf{d}$, Bright-field images of wild-type and Mmp14-knockout ES cell spheroids in Matrigel $48 \mathrm{~h}$ after 2i-LIF removal. $n=3$ independent experiments. e, Quantification of ES cell-spheroid area in wild-type and Mmp14-knockout ES cells. $n=607$ wild-type and 600
Mmp14-knockout spheroids. Two-sided unpaired Student's $t$-test; ${ }^{* * * *} P<0.0001$; mean \pm s.e.m. $\mathrm{f}$, Representative examples of control and MMP inhibitor $(100 \mu \mathrm{M} \mathrm{NSC} 405020+20 \mu \mathrm{M}$ prinomastat)-treated ES cells and XEN cells cultured in 2D. For ES cells, treatment started $48 \mathrm{~h}$ after 2i-LIF removal for $18 \mathrm{~h}$ as in b. XEN cells were treated with MMP inhibitors for $18 \mathrm{~h}$. MMP inhibitor treatment does not induce cell death in $2 \mathrm{D}$ cell cultures. $n=3$ independent experiments.g, Schematic showing the method for quantification of vector-map angles during embryo growth. $\mathbf{h}$, Representative example of an E5.75 embryo showing a vector map following the direction of perforations in the epiblast. $n=10$ embryos. $\mathbf{i}$, Quantification of vector angles presented as a frequency plot; each $15^{\circ}$ bin includes points of relative frequencies from 10 embryos. The same data are presented as a circular histogram in Fig. $4 \mathrm{~d}$. Kolmogorov-Smirnov test; ${ }^{* * * *} P<0.0001$. Centre lines show median values and box limits represent the maximum and minimum values. Scale bars, $20 \mu \mathrm{m}$. 


\section{Article}
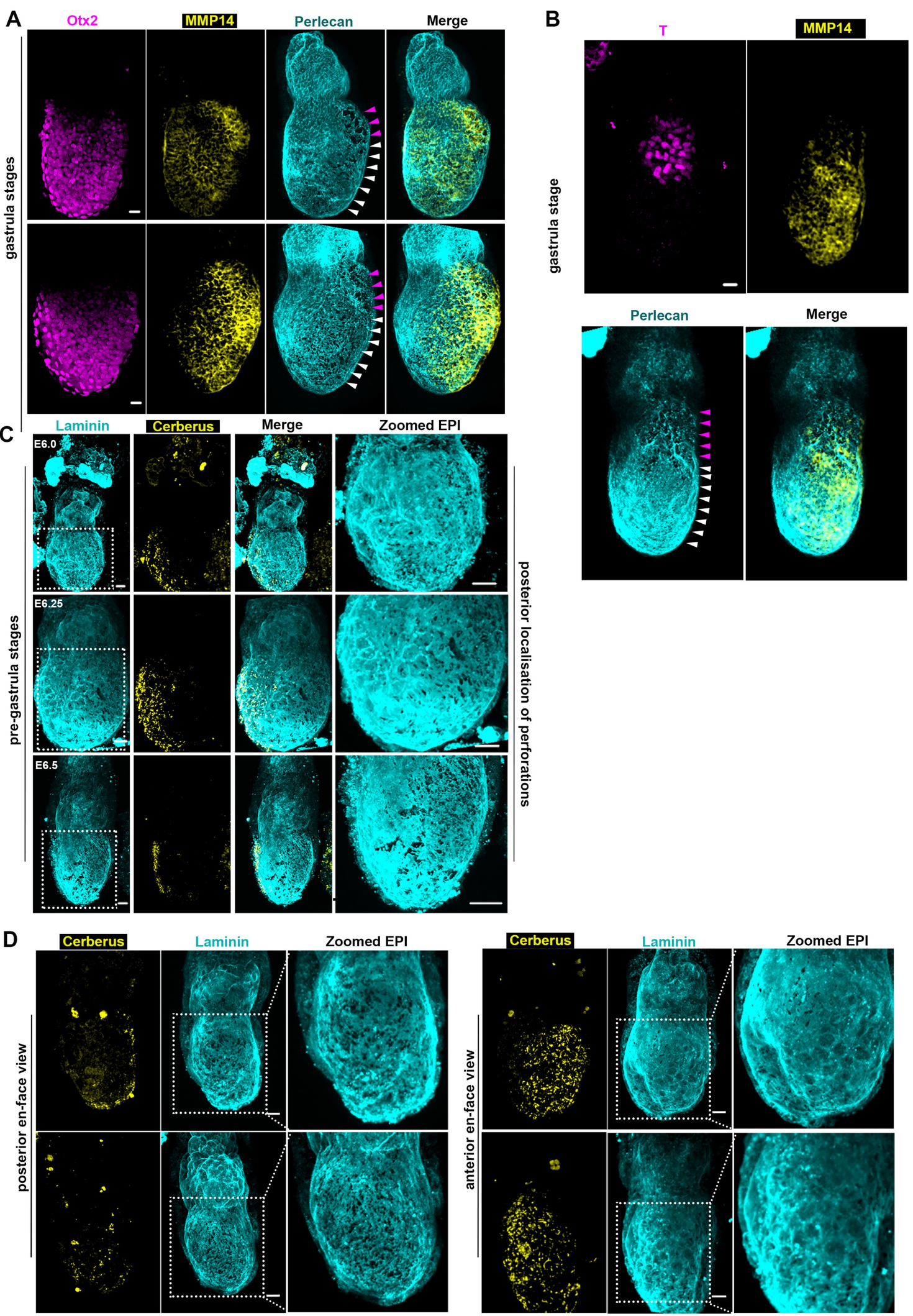

Extended Data Fig. 9 |Posterior localization of basement membrane perforations and MMP14 expression in pre-gastrula and gastrula stages. a, Representative examples of early-gastrula embryos showing posterior expression of MMP14. $n=15$ embryos. b, En face posterior view of early-gastrula embryo. $n=10$ embryos. In a, b, MMP14 expression follows the pattern of basement membrane perforations. White arrowheads, posterior and prospective primitive streak; purple arrowheads, breached basement membrane-primitive streak.c, Representative examples of E6.0-E6.5 embryos showing the pattern of basement membrane perforations in pre-gastrula stages. Basement membrane perforations pre-pattern the primitive streak in pre-gastrula stages. $n=30$ embryos. $\mathbf{d}$, En face posterior and anterior views of pre-gastrula embryos showing that basement membrane perforations are found only at the posterior side of the embryo. $n=10$ embryos. Scale bars, $20 \mu \mathrm{m}$. 

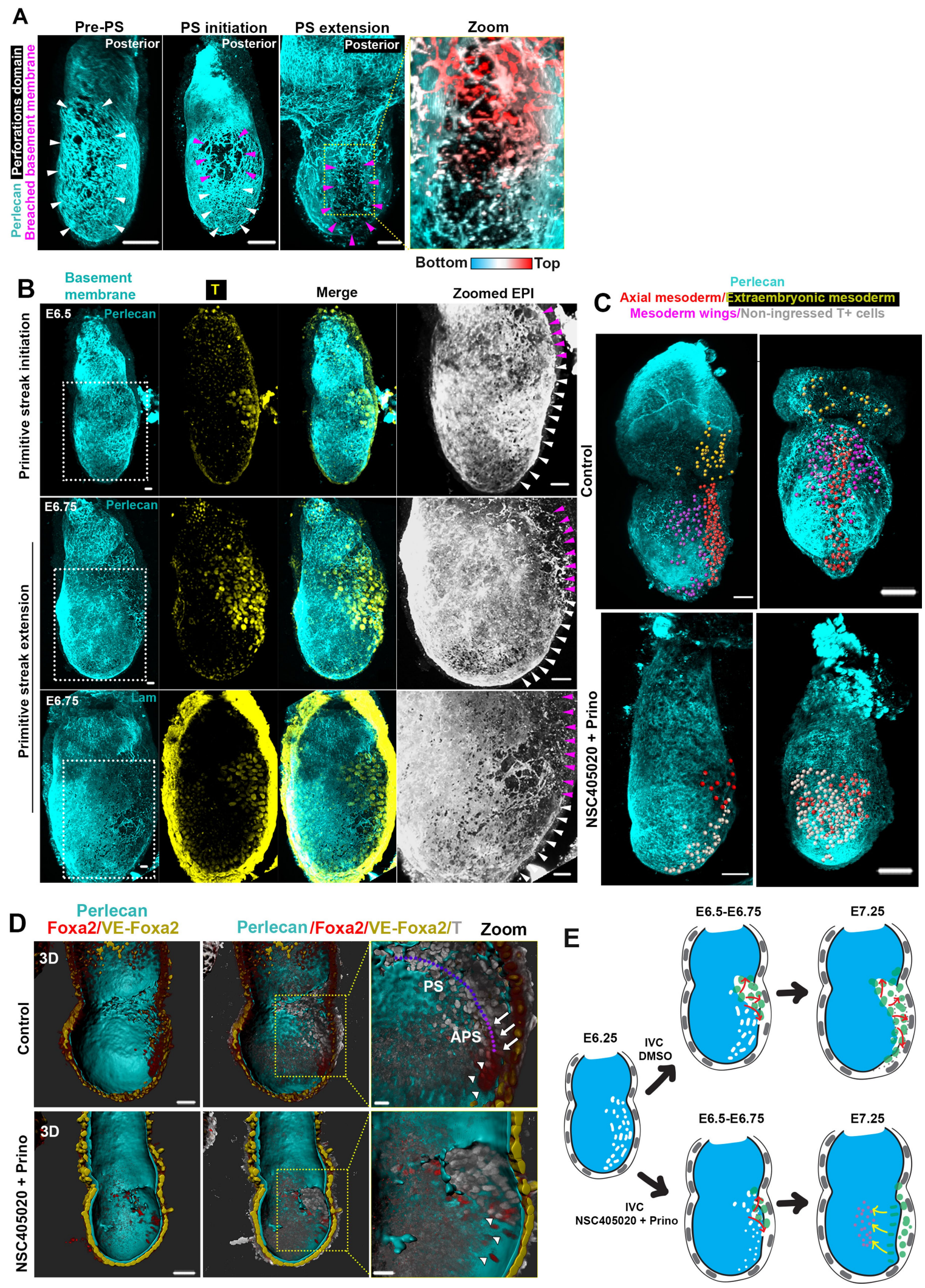

Extended Data Fig. 10 | See next page for caption. 


\section{Article}

Extended Data Fig.10 | MMP-mediated basement membrane remodelling regulates primitive-streak extension. a, Posterior en face views of gastrula embryos. Purple arrowheads, primitive streak (PS); white arrowheads, perforations. Right, depth-coded view. Epiblast basement membrane, cyan; endoderm basement membrane, white-red. $n=10$ embryos. Scale bars, $50 \mu \mathrm{m}$ b, Representative examples of gastrula-stage embryos showing the pattern of basement membrane perforation in correlation with $T$ (encoding Brachyury) expression (nuclear localization, halo around the embryo is due to background staining). White arrowheads, prospective primitive streak. Purple arrowheads, breached basement membrane-primitive streak. $n=20$ embryos. Scale bars $20 \mu \mathrm{m}$. c, Representative examples of embryos cultured (for $18 \mathrm{~h}$ ) from early
E6.5 (pre-primitive streak stage); $n=11$ control and 17 MMP inhibitor-treated embryos. Scale bars: $50 \mu \mathrm{m}$ (left), $80 \mu \mathrm{m}$ (right; Supplementary Videos 8, 9). d, Surface-rendered images of embryos cultured from early E6.5 (for 18 h). Foxa 2 is an anterior primitive streak (APS) marker. Ingressed (white arrows) and non-ingressed (white arrowheads) Foxa2-expressing cells. APS, magenta dotted line; primitive streak, purple dotted line. $n=10$ control and 10 MMP inhibitor-treated embryos. Scale bars, $80 \mu \mathrm{m}$ (main images); $20 \mu \mathrm{m}$ (enlarged area).e, Schematic representation of primitive-streak extension along the perforation path and interpretation of primitive-streak appearance after inhibition of MMPs (NSC405020 + prinomastat) just before gastrulation. 


\section{natureresearch}

\section{Reporting Summary}

Nature Research wishes to improve the reproducibility of the work that we publish. This form provides structure for consistency and transparency in reporting. For further information on Nature Research policies, see Authors \& Referees and the Editorial Policy Checklist.

\section{Statistics}

For all statistical analyses, confirm that the following items are present in the figure legend, table legend, main text, or Methods section.

n/a Confirmed

$\square \bigotimes$ The exact sample size $(n)$ for each experimental group/condition, given as a discrete number and unit of measurement

$\square$ \A statement on whether measurements were taken from distinct samples or whether the same sample was measured repeatedly

$\square$ The statistical test(s) used AND whether they are one- or two-sided

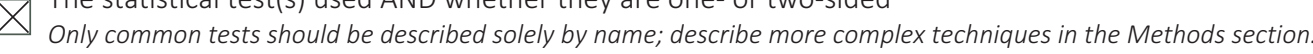

Х $\square$ A description of all covariates tested

Х $\square$ A description of any assumptions or corrections, such as tests of normality and adjustment for multiple comparisons

$\square$ A full description of the statistical parameters including central tendency (e.g. means) or other basic estimates (e.g. regression coefficient)

AND variation (e.g. standard deviation) or associated estimates of uncertainty (e.g. confidence intervals)

$\square$ For null hypothesis testing, the test statistic (e.g. $F, t, r$ ) with confidence intervals, effect sizes, degrees of freedom and $P$ value noted

$\square$ Give P values as exact values whenever suitable.

Х $\square$ For Bayesian analysis, information on the choice of priors and Markov chain Monte Carlo settings

Х $\square$ For hierarchical and complex designs, identification of the appropriate level for tests and full reporting of outcomes

Х $\square$ Estimates of effect sizes (e.g. Cohen's $d$, Pearson's $r$ ), indicating how they were calculated

Our web collection on statistics for biologists contains articles on many of the points above.

\section{Software and code}

Policy information about availability of computer code

Data collection

LEICA software LAS X (v. 3.5.6.21594) was used for image acquisition.

Data analysis

The code used in this study are available at https://github.com/darogan/Kyprianou_Zernika-Goetz and https://doi.org/10.5281/ zenodo.3610335.

For graphical statistics and statistical tests (t-test), GraphPad Prism 7.0a was used.

Fiji image processing software (v.1.52p) was used for image processing and analysis.

Imaris 9.0 image processing software was used for image processing and analysis.

Oriana Software (Version 4) was used for visualising circular statistics.

Re-analysis of the GSE109071(from 10.1016/j.celrep.2019.02.031) dataset was performed using R (v3.4.4) and Seurat (v3.0.1). ChIP tracks for Smad2_3 from GSE70486(from 10.1016/j.stem.2016.10.002) in TDF (tiled data file) format were visualised in IGV (Integrative Genomics Viewer, 10.1038/nbt.1754).

For manuscripts utilizing custom algorithms or software that are central to the research but not yet described in published literature, software must be made available to editors/reviewers. We strongly encourage code deposition in a community repository (e.g. GitHub). See the Nature Research guidelines for submitting code \& software for further information. 
Policy information about availability of data

All manuscripts must include a data availability statement. This statement should provide the following information, where applicable:

- Accession codes, unique identifiers, or web links for publicly available datasets

- A list of figures that have associated raw data

- A description of any restrictions on data availability

Scripts and data for single cell RNA-seq and Chip-seq analysis are available from GSE109071,GSE70486 and https://github.com/darogan/Kyprianou_Zernika-Goetz. The source data used in all the presented graphs are provided as a Source Data files. Raw image files are available from the corresponding author upon request.

\section{Field-specific reporting}

Please select the one below that is the best fit for your research. If you are not sure, read the appropriate sections before making your selection. $\bigotimes$ Life sciences $\quad \square$ Behavioural \& social sciences $\square$ Ecological, evolutionary \& environmental sciences

For a reference copy of the document with all sections, see nature.com/documents/nr-reporting-summary-flat.pdf

\section{Life sciences study design}

All studies must disclose on these points even when the disclosure is negative.

Sample size Sample size was determined based on our previous experience and the work of other groups using mouse embryos as experimental model systems.

(Cell. 2014 Feb 27; 156(5): 1032-1044)

(Nature Cell Biology volume 17, 113-122 (2015)

(Nature Cell Biology volume 18, 1281-1291 (2016)

(Nature Cell Biology volume 20, 1278-1289 (2018)

Data exclusions No data were excluded from the analysis

Replication In the study, all attempts at replication were successful.

Each result described in the paper is based on at least two independent biological replicates but very often an experiment is based on more than two experiments.

Randomization Samples (mouse embryos) were allocated randomly into experimental groups

Blinding The investigators were not blinded to group allocation due to the nature of embryo recovery. Thus, it was not possible to blind the experimenters when $\mathrm{i}$ treatments were performed. When assessing treatment result on basement membrane appearance, it was either quantified or judged simply on presence or absence of perforations.

\section{Reporting for specific materials, systems and methods}

We require information from authors about some types of materials, experimental systems and methods used in many studies. Here, indicate whether each material, system or method listed is relevant to your study. If you are not sure if a list item applies to your research, read the appropriate section before selecting a response.

Materials \& experimental systems

\begin{tabular}{l|l}
\hline n/a & Involved in the study \\
\hline & $\bigotimes$ Antibodies \\
$\square$ & $\bigotimes$ Eukaryotic cell lines \\
$\square$ & $\square$ Palaeontology \\
$\square$ & $\square$ Clinical data
\end{tabular}

\begin{tabular}{l|l} 
Methods \\
\hline n/a & Involved in the study \\
$\square$ & $\square$ ChIP-seq \\
$\searrow$ & $\square$ Flow cytometry \\
$\square$ & $\square$ MRI-based neuroimaging
\end{tabular}

\section{Antibodies}

Antibodies used

Primary Antibodies used in whole mount immunofluorescence:

E-cadherin (1:300; Thermofisher Scientific, 13-1900; lot: TD2496212 and 74960345A)

Oct4 (1:400; Santa Cruz Biotechnologies, sc-5279; lot: E1017 and E1818)

Laminin (1:400; Sigma, L9393; lot: 046M4837V)

Collagen IV (1:100; Millipore, AB769; lot: 2818807 and 3182110) 
HSPG2 (1:100; Millipore, MAB1948P; lot: 2890988 and 3094623)

Otx2 (1:100; R\&D Systems, AF1979; lot: KNO0615111 and KNO0616081)

Cerberus (1:500; R\&D Systems, MAB1986; cl. 225807; lot: JUP031904A)

Foxa2 (1:200; Cell Signalling Technologies, D56D6; lot: 3)

T/Brachyury (R\&D Systems, AF2085; lot: KQP0619031)

MMP14 (IF: 1:150; WB: 1:2000; Abcam, ab51074; cl. EP1264Y; lot: GR300562-16)

P-Histone H3 (S10) (1:300; Cell Signaling Technologies, 9701S; lot: 17)

Cleaved Caspase-3 (D175) (5A1E) (1:200; Cell Signaling Technologies, 9664S; lot: 21)

hAP-2gamma (1:200; R\&D Systems, AF5059; lot: CBHR011904A and CBHR0219051)

Secondary antibodies used:

Alexa 488 donkey anti-mouse (1:500; Thermo Fisher Scientific, A21202; lot: 2018296)

Alexa 594 donkey anti-rat (1:500; Thermo Fisher Scientific , A21209; lot: 1870948)

Alexa 647 donkey anti-rabbit (1:500; Thermo Fisher Scientific, A31573; lot: 1903516)

Alexa 488 donkey anti-goat (1:500; Thermo Fisher Scientific, A11055; lot: 1942238)

Validation

The subcellular localization of all the proteins analyzed in this study has been previously reported. This was used to validate the specificity of the antibody.

E-cadherin: it correctly stained the basolateral side of cells in the embryo as reported and as expected (Science 356, doi:10.1126/ science.aal1810)

Oct4: it specifically stained the epiblast at all stages tested, as expected (Science 356, doi:10.1126/science.aal1810)

Laminin: it correctly stained the basement membrane between visceral endoderm and Exe or epiblast, as reported elsewhere and as expected (Dev Dyn 241, 270-283)

Collagen IV: it correctly stained the basement membrane between visceral endoderm and Exe or epiblast, as reported elsewhere and as expected (Dev Dyn 241, 270-283)

HSPG2: same as above

Otx2: it was correctly expressed in the epiblast and embryonic visceral endoderm of post-implantation embryos (eLife 2018;7:e32839)

Cerberus: correctly stained anterior visceral endoderm in post-implantation mouse embryos (Dev Bio 309, 97-112)

Foxa2: it was correctly expressed in the visceral endoderm and in the gastrulation stage mesoderm (Development 136,

1029-1038)

T/Brachyury: it correctly stained mesoderm at 6.5 and later as reported and expected (Dev Biol 288, 363-371)

c-Casp3: correctly stained apoptotic cells as reported before (Autophagy, doi.org/10.1080/15548627.2019.1630222)

MMP14: expected membrane staining in the epiblast cells where MMP14 is expressed according to single cell sequencing analysis and confirmed by immunofluorescence and western blot in wildtype and MMP14 knockout ES cell lines in this study. AP2gamma: it correctly stained the ExE lineage in mouse postimplantation embryos as reported and expected (Genes Dev 29(23):2435-48.

Anti phospho histone 3 it correctly labelled mitotic cells, as expected (Science, 360(6384):99-102 2018)

\section{Eukaryotic cell lines}

Policy information about cell lines

Cell line source(s)

Lifeact-GFP mESCs were derived from the Lifeact-GFP transgenic mouse line (Reidl, J. et al., 2010).

MMP14-KO mESCs were generated in this study in Lifeact-GFP mESCs.

E14 WT mESCs (kindly provided by Prof. Austin Smith, Stem Cell Institute, Cambridge, UK)

Mouse extraembryonic endoderm cells: EGFP XENs (a gift from Dr. Peter Rugg-Gunn Babraham Institute, Cambridge).

Authentication

Cells were maintained in conditions to preserve stem cell character and prevent differentiation. The self-renewal properties of WT ESCs were confirmed by immunofluorescence routinely and morphological characterstics. WT ESC colonies maintained a domed morphology and XEN cells showed a mixed morphology of fibroblast-like and rounded cells as expected.

Mycoplasma contamination

Cell lines were routinely tested for mycoplasma contamination by PCR and confirmed that they were negative for mycoplasma contamination.

Commonly misidentified lines (See ICLAC register)

No commonly misidentified cell lines were used

\section{Animals and other organisms}

Policy information about studies involving animals; ARRIVE guidelines recommended for reporting animal research

Laboratory animals

6-week old mice (Mus musculus) were used to obtain mouse embryos for this study. The following strains and geneticallymodified models were used: F1 (C57Bl6xCBA), MF1, CD1, Nodal fl/fl; RCre:T2, TTR:Cre; RhoA fl/fl and T:GFP (both males and females).

Wild animals

The study did not involve wild animals.

Field-collected samples

The study did not involve samples collected from the field. 
